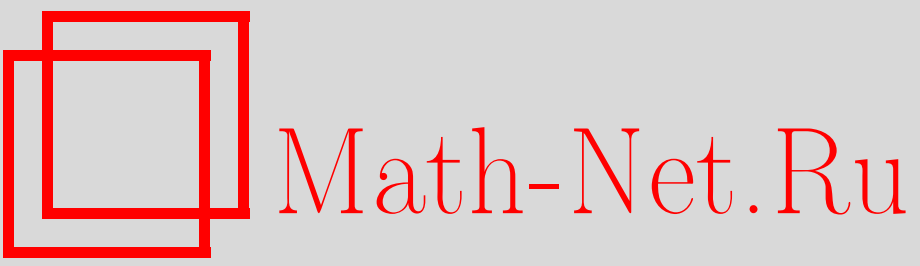

Ю. М. Важенин, А. Г. Пинус, Элементарная классификация и разрешимость теории производных структур, УМH, 2005, том 60, выпуск 3, 3-40

DOI: https://doi.org/10.4213/rm1428

Использование Общероссийского математического портала Math-Net.Ru подразумевает, что вы прочитали и согласны с пользовательским соглашением

http://www.mathnet.ru/rus/agreement

Параметры загрузки:

IP: 54.166 .219 .16

26 апреля 2023 г., 15:03:14 


\title{
ЭЛЕМЕНТАРНАЯ КЛАССИФИКАЦИЯ И РАЗРЕШИМОСТЬ ТЕОРИЙ ПРОИЗВОДНЫХ СТРУКТУР
}

\author{
Ю.М. ВАЖЕНИН, А. Г. ПИНУС
}

В работе приводится обзор результатов, связанных с элементарньпи теориями производных структур классических и универсальных алгебр. Сформулирован ряд открытых вопросов.

Библиография: 129 названий.

\section{СОДЕРЖАНИЕ}

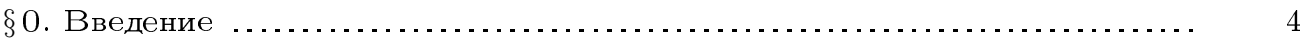

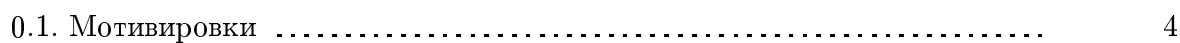

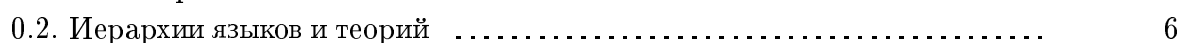

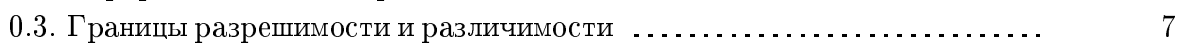

0.4. Метод интерпретации ...................................... 11

$\S 1$. Линейные преобразования векторных пространств и модулей ....... 11

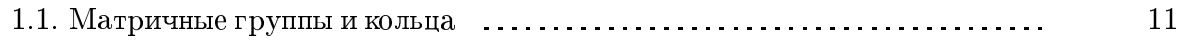

1.2. Полугруппы эндоморфизмов и группы автоморфизмов бесконечномерных

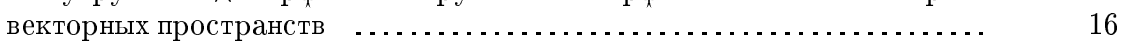

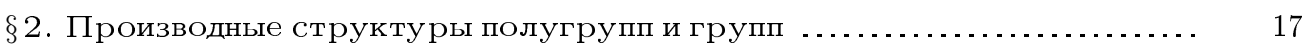

2.1. Решетки подполугрупп и подгрупп ............................. 17

2.2. Решетки конгруэнций и близкие к ним производные структуры . . . . . . . . 19

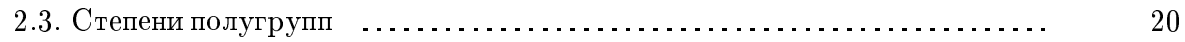

$\S 3$. Преобразования упорядоченных множеств и графов ................ 20

3.1. Полугруппы эндоморфизмов и направленных преобразований $\ldots . . . . .20$

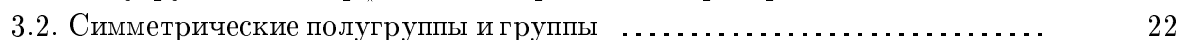

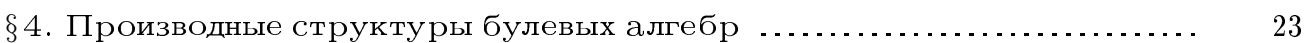

4.1. Группы автоморфизмов булевых алгебр ........................ 23

4.2. Решетки конгруэнций и подалгебр булевых алгебр .................. 26

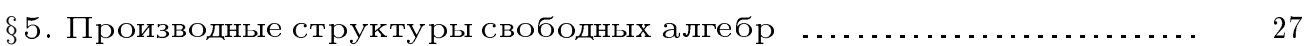

$\S 6$. Производные структуры топологических пространств ............. 33

Список литературы ............................................. 35

Работа выполнена при финансовой поддержке РФФИ (грант № 02-01-00258). 


\section{$\S$ 0. Введение}

0.1. Мотивировки. Производной структурой данной алгебраической системы $\mathfrak{A}$ называется алгебраическая система $D(\mathfrak{A})$, однозначно определяемая по $\mathfrak{A}$ и несушая в себе ту или иную информацию о строении системы $\mathfrak{A}$. Для произвольных алгебраических систем $\mathfrak{A}$ в качестве их производных структур традиционно и всесторонне рассматривались группы автоморфизмов $\operatorname{Aut}(\mathfrak{A})$, моноиды эндоморфизмов $\operatorname{End}(\mathfrak{A})$, peшетки подсистем $\operatorname{Sub}(\mathfrak{A})$, решетки конгруэнций $\operatorname{Con}(\mathfrak{A})$, полугруппы внутренних изоморфизмов Iso $\mathfrak{A}$ и т. п. В более частных ситуациях в качестве производных структур изучались и другие, связанные со спецификой алгебраической системы $\mathfrak{A}$, производные алгебраические системы $D(\mathfrak{A})$ : для колец (полей) $\mathfrak{A}$ - всевозможные матричные кольца над $\mathfrak{A}$, для графов (упорядоченных множеств) - различные полугрупшы их преобразований и т. д.

Свое начало изучение производных структур берет еше в XIX веке, и соответствующая тематика присутствует ньне практически во всех разделах алгебры, занимая в них центральные места. Более того, идея введения и исследования производных структур имеет общематематическое значение. В самом деле, такие структуры возникают не только в связи с изучением алгебраических систем, но и при рассмотрении объектов иного рода в самых разных областях математики. Упомянем, например, фундаментальные группы и алгебры замыканий топологических пространств, полугрупшы непрерывных линейных операторов банаховых пространств, синтаксические моноиды кодов и т. д. При этом часто возникают плодотворные взаимосвязи между различньми областями математики: топологические и иные системы изучаются с помощью алгебраических производных структур алгебраическими методами, а алгебраические системы - с помошю топологических и иных систем (к примеру: булевы алгебры - с помощью их стоуновских пространств).

Производная структура $D(\mathfrak{A})$ данного типа $D$ обычно несет существенную информацию о системе $\mathfrak{A}$ и доставляет новый, нередко достаточно удобньй язык, на котором можно исследовать строение системы $\mathfrak{A}$. Этим объясняется широта круга вопросов, связанных с рассмотрением взаимосвязей свойств исходной алгебраической системы $\mathfrak{A}$ и соответствующей производной структуры $D(\mathfrak{A})$. Образцы исследования таких вопросов содержатся во многих работах, среди которых упомянем, например, труды Р. Бэра [1], Ж. Лаллемана [49], А. Г. Пинуса [80], Б. И. Плоткина [86], М. Судзуки [105], Д. А. Супруненко [106], Л. Н. Шеврина и А.Я. Овсянникова [102].

Одним из основных вопросов, изучаемых в указанных и многих других публикашиях, является вопрос определимости системы $\mathfrak{A}$ своей производной структурой $D(\mathfrak{A})$ : следует ли из изоморфизма $D(\mathfrak{A}) \cong D(\mathfrak{B})$ производных структур данного типа $D$ изоморфизм (или иная, достаточно тесная связь, см., к примеру, [78]) систем $\mathfrak{A}$ и $\mathfrak{B}$ ? Поскольку утвердительный ответ на этот вопрос встречается относительно редко, обычно рассматривается следуюший вопрос: найти необходимые и достаточные условия на алгебраические системы $\mathfrak{A}$ и $\mathfrak{B}$, при которых имеет место изоморфизм производных систем $D(\mathfrak{A})$ и $D(\mathfrak{B})$. Оба этих вопроса можно трактовать как задачу классификации исходных систем с точностью до изоморфизма производных структур muna $D$.

Развитие математической логики и теории моделей вызвало к жизни цельй ряд важных и естественных проблем. Одной из центральных среди них является проблема әлементарной классификации, т.е. классификации алгебраических систем с 
точностью до совпадения их теорий в языке логики первого порядка. Актуальность указанной проблемы убедительно и вьпукло продемонстрирована в книгах Ю. Л. Ершова [31], Ю. Л. Ершова и Е. А. Палютина [33]. Применив к производным структурам понятие элементарной эквивалентности, мы получаем обшую формулировку проблемы элементарной классификачии производных структур:

найти необходимые и достаточные условия на алгебраические системы $\mathfrak{A}$ и $\mathfrak{B}$ данного класса, при которых имеет место элементарная эквивалентность $D(\mathfrak{A}) \equiv$ $D(\mathfrak{B})$ их производных структур типа $D$.

Другим важным вопросом, связанным, в частности, с элементарной классификацией, яВляется проблема разрешимости теорий первого порядка производных струкmyp:

существует ли алгоритм, которьй по любому предложению $\varphi$ сигнатуры производной структуры $D(\mathfrak{A})$ из данного язька $L$ (фрагмента языка первого порядка) отвечает на вопрос об истинности $\varphi$ на системе $D(\mathfrak{A})$, другими словами, разрешима ли теория $L D(\mathfrak{A})$ ?

Обзор и систематизация результатов по этим двум и близким к ним проблемам и является целью нашей работы.

Заметим также, что с проблемой элементарной классификации производных структур связаны понятия чисел Левенгейма и Ханфа. Напомним, что числом Левенгейма (Ханфа) для класса $K$ алгебраических систем назьвается наименьший кардинал $k$ такой, что для любой системы $\mathfrak{A} \in K$ существует элементарно эквивалентная системе $\mathfrak{A}$ система $\mathfrak{A}^{\prime} \in K$ такая, что $\left|\mathfrak{A}^{\prime}\right| \leqslant k$ (если $|\mathfrak{A}| \geqslant k$, то сушествуют $K$-системы, элементарно эквивалентные системе $\mathfrak{A}$, произвольно большой мощности). В случае аксиоматизируемости класса $K$ в логике первого порядка в силу теоремы Левенгейма-Сколема-Тарского об элементарных подсистемах и элементарных расширениях числа Левенгейма и Ханфа для $K$ совпадают и равны $\aleph_{0}$. В случае же неаксиоматизируемости класса $K$, что чаше всего имеет место при изучении классов производных структур, числа Левенгейма и Ханфа дают существенную информашию о строении отношения элементарной эквивалентности на классе производных структур. Мы слегка подкорректируем определения чисел Левенгейма и Ханфа при работе с классами $D(K)=\{D(\mathfrak{A}) \mid \mathfrak{A} \in K\}$ производных структур. D-числом Левенгейма (Ханфа) для класса $K$ назовем наименьший кардинал $k$ такой, что для любой системы $\mathfrak{A} \in K$ сушествует система $\mathfrak{A}^{\prime} \in K$ такая, что $D(\mathfrak{A}) \equiv D\left(\mathfrak{A}^{\prime}\right)$ и $\left|\mathfrak{A}^{\prime}\right| \leqslant k$ (если $|\mathfrak{A}| \geqslant k$, то сушествуют $K$-системы $\mathfrak{A}^{\prime}$ как угодно большой мошности такие, что $\left.D(\mathfrak{A}) \equiv D\left(\mathfrak{A}^{\prime}\right)\right)$. Очевидно, что мошности элементарно $D$-определимых $K$-систем не превышают минимального из $D$-чисел Левенгейма и Ханфа для класса $K$.

Работа над данным обзором была начата авторами в 2000 г. Неожиданный, скоропостижный уход из жизни Ю. М. Важенина в 2003 г. на некоторое время прервал эту работу. Тем не менее второй автор считает своим долгом завершить задуманное и посвятить эту работу памяти Юрия Михайловича - прекрасного человека, известного алгебраиста. Хочу также выразить свою благодарность Ю. В. Нагребецкой, О. В. Белеградеку и В. А. Толстых за помошь в работе над разделами 1.1 и 1.2. 
0.2. Иерархии языков и теорий. Для уточнения формулировок рассматриваемых вопросов определим понятия иерархий языков и теорий первого порядка. Они были введены первым из авторов обзора в [121] и подвергнуты детальному исследованию в его работах [122], [123].

Пусть $\mathscr{E}$ - совокупность всех формул логики первого порядка фиксированной сигнатуры $\sigma$, записанных в пренексной нормальной форме. Языком сигнатуры $\sigma$ будем называть любое подмножество множества $\mathscr{E}$. Само $\mathscr{E}$ назовем әлементарнылм языком. Пусть $Q_{i} \in\{\forall, \exists\}, Q_{i} \neq Q_{i+1}$ для $i \in\{1, \ldots, p-1\}$ и $r, s, t \in\{0,1\}$. Определим язык $Q_{1} \ldots Q_{p} \neg^{r} \wedge^{s} \vee^{t}$, где $z^{1}=z$ и $z^{0}-$ пустой символ для $z \in\{\neg, \wedge, \vee\}$, следуюшим образом. Во-первых, блочная схема кванторной приставки каждой из формул языка $Q_{1} \ldots Q_{p} \neg^{r} \wedge^{s} \vee^{t}$ является подсловом слова $Q_{1} \ldots Q_{p}$. Во-вторых, связка $\neg, \wedge, \vee$ допускается в бескванторной части $\xi$ этих формул, если соответственно $r=1, s=1, t=1$, и не допускается, если соответственно $r=0, s=0, t=0$. Обозначим, кроме того, через $\neg^{r} \wedge^{s} \vee^{t}$ бескванторный подъязьк язька $\forall \neg^{r} \wedge^{s} \vee^{t}$, через $\bar{\omega} \neg^{r} \wedge^{s} \vee^{t}$ - объединение $\bigcup_{p \in \omega} Q_{1} \ldots Q_{p} \neg^{r} \wedge^{s} \vee^{t}$, и пусть $\theta=\neg \wedge \vee$. Семейство $S A$ всех языков вида $Q_{1} \ldots Q_{p} \neg^{r} \wedge^{s} \vee^{t}, \neg^{r} \wedge^{s} \vee^{t}, \bar{\omega} \neg^{r} \wedge^{s} \vee^{t}$ упорядочивается включением и называется схемно-альтернативной иерархией языков. Более бедной по сравнению с этой иерархией языков является схемная иерархия, т.е. семейство $S$ всех язьков вида $Q_{1} \ldots Q_{p} \theta$ и $\bar{\omega} \theta$, упорядоченное включением. Пусть $n \leqslant \omega, \bar{n} \theta=\left\{Q_{1} \vec{x} \ldots Q_{p} \vec{y} \xi \mid p \leqslant n\right\}$. Переменной иерархией назьвается упорядоченное включением семейство языков $V=\{\bar{n} \theta \mid n \leqslant \omega\}$. Наконец, альтернативной и переменно-альтернативной иерархией назьваются семейства $A=\left\{\bar{\omega} \neg^{r} \wedge^{s}\right.$ $\left.\vee^{t} \mid r, s, t \in\{0,1\}\right\}$ и $V A=\{X \cap Y \mid X \in V, Y \in A\}$ соответственно. Тривиальной иерархией назьвается одноэлементное семейство $\{\mathscr{E}\}$.

Вхождение формулы $\varphi$ логики первого порядка в некоторый язьк в рамках той или иной рассматриваемой иерархии говорит о сложности этой формулы в том или ином, специфическом для данной конкретной иерархии, смысле.

Пусть теперь $K$ - некоторьй класс алгебраических систем фиксированной сигнатуры $\sigma$ и $L-$ некоторый язык логики первого порядка. Теорией класса $K$, ограниченной языком $L$ ( $L$-теорией класса $K)$, называется совокупность $L K$ всех тех предложений $\varphi$ из $L$, которые истинны на каждой системе из $K$ :

$$
L K=\{\varphi \in L \mid \varphi \text {-предложение и для любой } \mathfrak{A} \in K \text { имеет место } \mathfrak{A} \models \varphi\} .
$$

Если теперь $H$ - одна из определенных вьше иерархиий языков логики первого порядка, то через $H K$ обозначим соответствующую иерархию теорий класса $K$, т.е. упорядоченную отношением теоретико-множественного включения совокупность

$$
H K=\{L K \mid L \in H\}
$$

Цельй ряд языков из самой богатой схемно-альтернативной иерархии хорошо известны и широко исследовались. Среди них: элементарный язык $\mathscr{E}$, позитивный $\bar{w} \wedge \vee$, әкзистенциальный $\exists \theta$, примитивный $\exists \neg \wedge$, сколемовский $\forall \exists \theta$, диофантов или, по другой терминологии, позитивно-примитивный $\exists \wedge$, универсальный $\forall \theta$ и эквациональныц $\forall$ языки. Для четырех из них обычно используются следуюшие обозначения:

$$
\bar{w} \wedge \vee=\mathscr{P}, \quad \exists \wedge=\mathscr{D}, \quad \forall \theta=U, \quad \forall=I .
$$


Названия языков переносятся на названия соответствуюших теорий класса $K$. В связи с этим говорят об элементарной, позитивной, экзистенциальной, примитивной, сколемовской, диофантовой, универсальной и эквациональной теориях данного класса $K$ алгебраических систем.

Представим диаграммы рассматриваемых иерархий языков логики первого порядка. Диаграммы тривиальной и схемной иерархии устроены предельно просто: первая одноэлементна, вторая - цеп типа $\omega+1$. Диаграммы схемной, альтернативной, переменно-альтернативной и схемно-альтернативной иерархий язьков представлены на рис. $1-4$.

0.3. Границы разрешимости и различимости. Сформулированные в разделе 0.1 проблемы $L$-классификации и разрешимости теорий производных структур зависят от рассматриваемого язька $L$, фиксируя который мы получаем формулировки конкретных проблем классификации и разрешимости. Так, например, возникает проблема элементарной классификации линейных групп и проблема разрешимости позитивной теории симметрической групшы над счетным множеством. Такого сорта проблемы активно исследовались в 60-50-х годах XX века (см., например, обзоры [32], [48]). Накопленная здесь к середине 80-х годов информация сделала актуальной задачу нахождения границы разрешимости [48], корректная постановка которой в терминах критических теорий была впервые опубликована в работе [121].

Определенные в предыдушем разделе иерархии позволяют предельно точно сформулировать проблему нахождения границы разрешимости, решение которой дает описание всех, в рамках данной иерархии, разрешимых теорий рассматриваемого класса алгебраических систем. Этот же "иерархический” подход дает возможность сформулировать и проблему нахождения границы различимости, решение которой дает описание всех языков $L$ из данной иерархии, которые различают алгебраические системы из рассматриваемого класса и для которых, стало быть, резонно исследовать проблему $L$-классификации. Приступим к формулировке указанных двух проблем.

Напомним, что теория $L K$ называется разрешимой, если существует алгоритм, которьй по любому предложению $\varphi \in \mathscr{E}$ отвечает на вопрос о принадлежности $\varphi$ теории $L K$. В противном случае, т.е. когда такого алгоритма не сушествует, теория $L K$ называется неразрешимой.

Пусть $H$ - одна из введенных в предыдущем разделе иерархий язьков и $H K$ - соответствующая иерархия теорий класса $K$ алгебраических систем.

ОПРЕДЕЛЕНИЕ. Теория $L K \in H K$ называется $H$-критической, если она является минимальной в иерархии $H K$ неразрешимой теорией. $H$-границей разрешимости класса $K$ назьвается множество

$$
B_{H}(K)=\{L \in H \mid L K-H \text {-критическая теория }\}
$$

Поскольку, как легко понять, для любой из наших иерарахий разрешимость наследуется для подтеорий, а неразрешимость - для надтеорий, а сами иерархии фундированы (не содержат бесконечно убьваюших последовательностей языков), то описание гранищы разрешимости $B_{H}(K)$ равнозначно описанию в рамках иерархии $H K$ всех разрешимых теорий класса $K$. Действительно, теория $L K \in H K$ разрешима тогда и только тогда, когда она не включает ни одну из теорий, входящих в $B_{H}(K)$. 


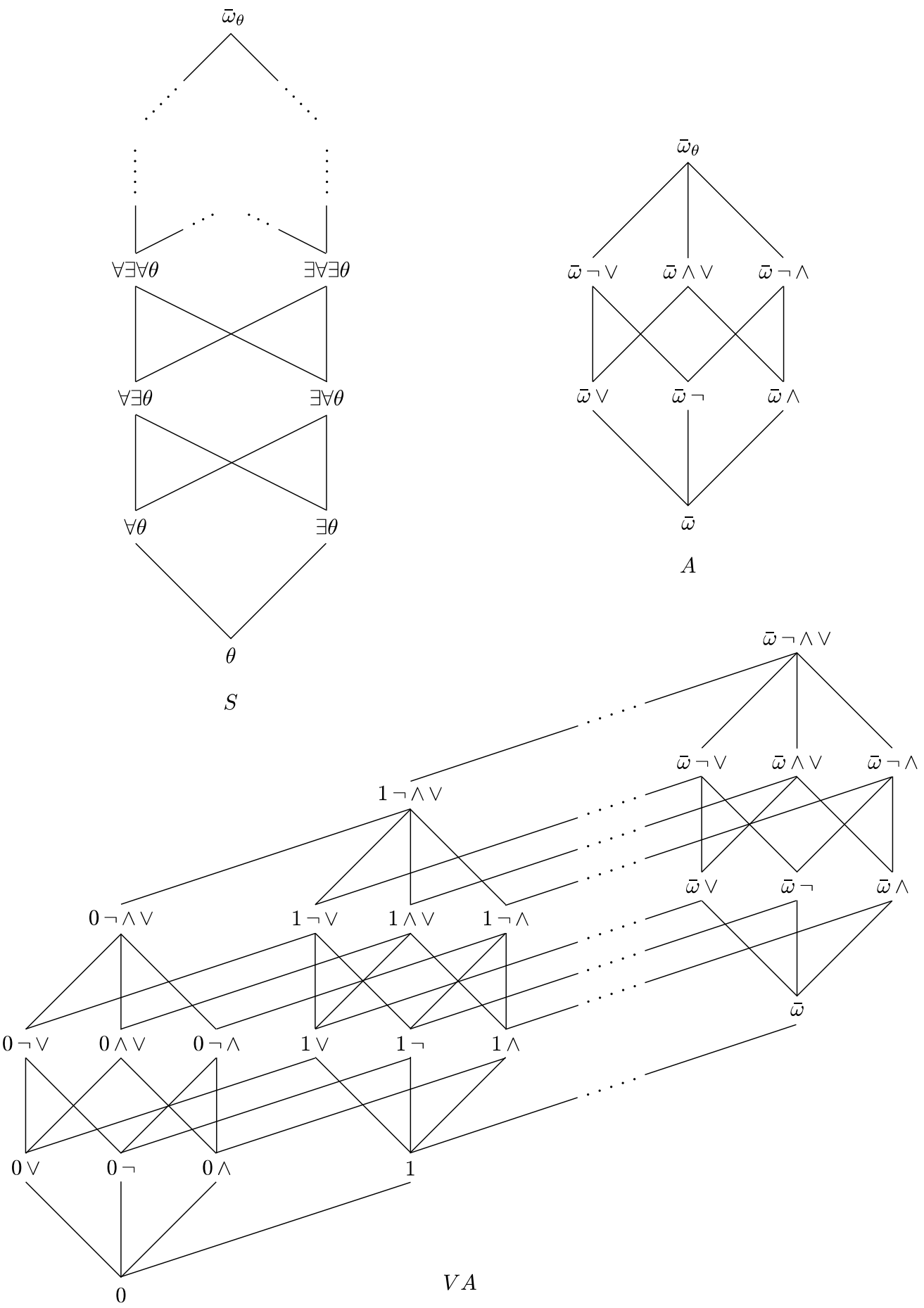

Рис. 1 

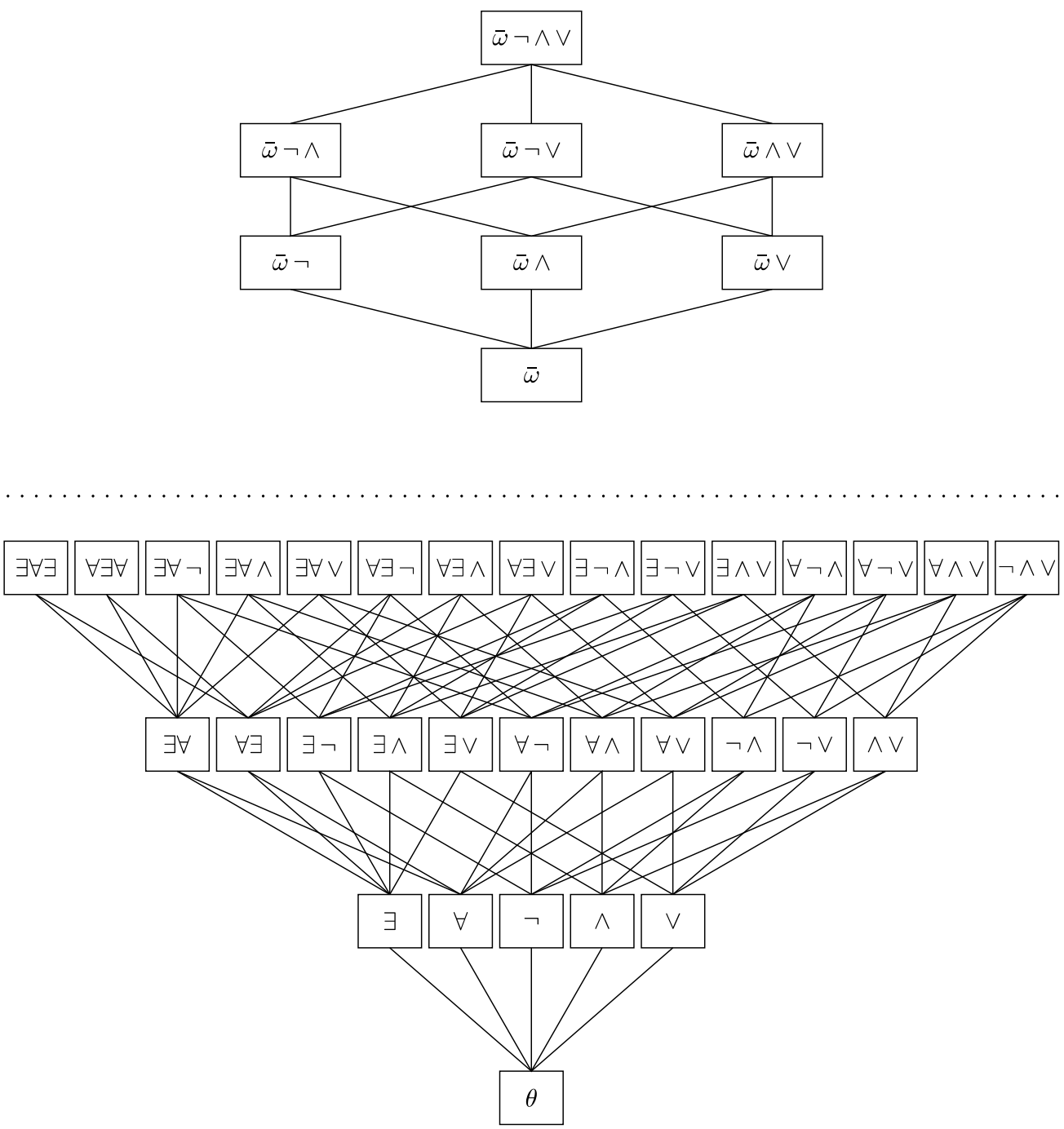

$S A$

Рис. 2

Отношение совпадения $L$-теорий алгебраических систем из класса $K$ задает естественное отношение эквивалентности ${ }_{L}$ на классе $K$ :

$$
\mathfrak{A} \equiv{ }_{L} \mathfrak{B} \text { тогда и только тогда, когда } L \mathfrak{A} \equiv L \mathfrak{B} .
$$

При этом каждая из рассматриваемых иерархий $H$ определяет на классе $K$ двойственную иерархию ${ }_{H}$ отношений эквивалентности

$$
\equiv_{H}=\left\{\equiv_{L} \mid L \in H\right\} \text {. }
$$

Через $\triangle_{K}$ и $\nabla_{K}$, как обычно, обозначим отношения изоморфизма и коллапса на классе $K$. 
ОПРЕДЕЛЕНИЕ. Язык $L$ иерархии языков $H$ назовем $K$-различающ, $u$, если отношение $\equiv_{L}$ на $K$ отлично от отношения $\nabla_{K}$, иначе говоря, если сушествуют системы $\mathfrak{A}, \mathfrak{B}$ из $K$ такие, что $L \mathfrak{A} \neq L \mathfrak{B}$. Систему $\mathfrak{A}$ назовем $L$-определимой в классе $K$, если класс $\equiv{ }_{L}$-эквивалентности системы $\mathfrak{A}$ в $K$ состоит только из систем, изоморфных $\mathfrak{A}$, т.е. если для любой системы $\mathfrak{B}$ из $K$ равенство $L \mathfrak{B}=L \mathfrak{A}$ влечет изоморфизм $\mathfrak{B} \cong \mathfrak{A}$. Систему $\mathfrak{A}$ из $K$ назовем сильно $L$-определимой, если существует $L$-предложение $\varphi$ такое, что для любой $K$-системы $\mathfrak{B}$ условия $\mathfrak{B} \models \varphi$ и $\mathfrak{B} \cong \mathfrak{A}$ равносильны. Минимальный в иерархии языков $H K$-различаюший язык назовем пограничнымм $K$-различающим языком; $H$-границей различимости для класса $K$ назьвается множество

$$
N_{H}(K)=\{L \in H \mid L-\text { пограничньй } K \text {-различающий язьк }\} \text {. }
$$

Для каждой из введенных иерархий язьков свойство “быть $K$-различающим" наследуется для надъязыков, а свойство "не быть $K$-различающим" наследуется подъязыками. Стало быть, язык $L \in H$ является $K$-различаюшим тогда и только тогда, когда он включает в себя хотя бы один из языков $H$-границы различимости $N_{H}(K)$ класca $K$.

В рамках данного обзора нас будут интересовать известные результаты и открытые проблемы, связанные с вопросами $L$-разрешимости, $L$-различимости, определения $H$-границ разрешимости, $H$-границ различимости, $L$-определимости, сильной $L$-определимости производных структур типа $D$ в классах $D(K)=\{D(\mathfrak{A}) \mid \mathfrak{A} \in K\}$ для различных естественных классов $K$ алгебраических систем, т.е. изучение возможностей использования элементарных теорий и их фрагментов для $D$-производных структур, с целью характеризации $K$-систем того либо иного класса $K$ алгебраических систем. К примеру, описание отношения эквивалентности $\equiv_{D}^{L}$, индуцированного на классе $K$ отношением $\equiv_{L}$ на классе $D(K)$ :

$$
\mathfrak{A} \equiv \stackrel{L}{D} \mathfrak{B} \Leftrightarrow D(\mathfrak{A}) \equiv{ }_{L} D(\mathfrak{B})
$$

описание в тех или иных приемлемых терминах $K$-систем, $D$-производные структуры которых являются $L$-определимыми, строго $L$-определимыми в классе $D(K)$, и пр. Большинство известных результатов, допускающих интерпретацию в рамках данной проблематики, касаются различных классов $K$ колец, полугрупп, векторных пространств, графов и упорядоченных множеств, булевых алгебр, свободных алгебр различных многообразий и др.

Заметим, наконец, что введенные вьше иерархии языков не содержат бесконечных несравнимых последовательностей (на самом деле их ширина не превьшает числа 16). В силу чего для любой из этих иерархий $H$ любые $H$-гранищы разрешимости и различимости суть конечные совокупности языков $H$-иерархии и, значит, допускают возможность явного описания.

Важной целью обзора, имеющей, по нашему мнению, методологическое значение, является “иерархический” взгляд на уже опубликованные результаты и унификация соответствуюшей теоминологии. Движение к этой цели позволяет по-новому осмыслить давно известные проблемы и открыть продуктивные пути для их дальнейшего развития и исследования. 
0.4. Метод интерпретации. Доказательство большинства рассмотренных далее результатов основано на использовании хорошо известного в своих самых различных вариациях метода интерпретации одного класса $K_{1}$ алгебраических систем с помощью некоторого языка $L$ в другом классе $K_{2}$. Мы остановимся здесь лишь на самой простой формулировке этого понятия, отсылая за точными определениями различных вариаций понятий интерпретации к специальной литературе по теории моделей [31], [32].

Пусть $K_{1}$ - это некоторьй класс универсальных алгебр чисто функциональной сигнатуры $\sigma_{1}=\left\langle f_{1}^{n_{1}}, \ldots, f_{r}^{n_{r}}\right\rangle$ без констант, а $K_{2}$ - класс универсальных алгебр сигнатуры $\sigma_{2}=\left\langle g_{1}^{m_{1}}, \ldots, g_{l}^{m_{l}}\right\rangle$ с теми же ограничениями на $\sigma_{2}$. Эти ограничения на сигнатуру рассматриваемых алгебраических систем несушественны и вызваны только целью упрощения формулировок. Пусть $L-$ некоторьй элементарньй язык. Тогда $к л а с c K_{2}$ допускает $L$-интерпретацию в классе $K_{1}$, если сушествуют $L$-формулы

$$
\phi_{1}\left(x_{1}, \ldots, x_{m_{1}}, y\right), \ldots, \phi_{l}\left(x_{1}, \ldots, x_{m_{l}}, y\right)
$$

сигнатуры $\sigma_{1}$ такие, что для любой $K_{1}$-алгебры $\mathfrak{A}=\left\langle A ; \sigma_{1}\right\rangle$ алгебра $\mathfrak{A} \phi=\left\langle A ; \sigma_{2}\right\rangle$ принадлежит классу $K_{2}$ и любая $K_{2}$-алгебра $\mathfrak{B}=\left\langle B ; \sigma_{2}\right\rangle$ изоморфна алгебре вида $\mathfrak{A} \phi$ для некоторой $K_{1}$-алгебры $\mathfrak{A}$. Здесь сигнатурные функции алгебры $\mathfrak{A} \phi$ определяются на множестве $A$ с помошью $L$-формул $\phi_{1}, \ldots, \phi_{l}$.

В случае, когда сушествуют $L$-интерпретации как класса $K_{1}$ в $K_{2}$, так и класса $K_{2}$ в $K_{1}$, будем говорить о $L$-биинтерпретируемости классов $K_{1}$ и $K_{2}$ друг в друге.

Подобная "строгая" формулировка понятия интерпретируемости чаше всего применима при изучении отношения $L$-эквивалентности $\equiv_{L}$ на различных классах алгебраических систем. В исследовании вопросов разрешимости (при доказательстве неразрешимости) чаше применяется понятие относительной әлементарной определимости одного класса систем в другом (родственное понятию интерпретации), описанное, к примеру, в монографии Ю. Л. Ершова [31].

\section{§1. Линейные преобразования векторных пространств и модулей}

1.1. Матричные группы и кольца. Исторически первыми результатами, касающимися элементарных теорий производных структур, были результаты о группах линейных преобразований векторных пространств и модулей над ассоциативными кольцами, т.е. результаты, касаюшиеся элементарных теорий матричных групा. Пусть $R$-некоторое ассоциативное кольцо с единицей. Здесь и далее через $G L(k, R)$ и $S L(k, R)$ обозначены общая линейная и специальная линейная групшы матриц порядка $k$ над кольцом $R$; через $U T(k, R)$ - подгруппа унитреугольных матриц группы $G L(k, R)$; через $M(k, R)$ - кольцо всех матриц порядка $k$ над $R$; наконец, через $N T(k, R)$ - кольцо нильтреугольных матриц из $M(k, R)$. Для кольца $R$ через $R^{*}$ обозначим двойственное к нему кольцо.

Первой работой по элементарным свойствам производных структур явилась работа А. И. Мальцева [53], в которой исследовались элементарные теории матричных групп над полями и кольцом $\mathbb{Z}$ целых чисел.

Теорема 1.1.1 (А.И. Мальцев [53]). Для любых полей $F, P$ нулевой характеристики и любьх натуральных чисел $k$ и $l$ следующие условия әквивалентны:

(1) $G L(k, F) \equiv G L(l, P)($ npu $k, l \geqslant 2)$; 
(2) $S L(k, F) \equiv S L(l, P)($ npu $k, l \geqslant 3)$;

(3) $F \equiv P$ u $k=l$.

Иначе говоря, в әлементарных теориях групп $G L(k, F)$ и $S L(k, F)$ выразимы u само поле $F$, и размер матрии, $k$.

Теорема 1.1 .2 (А. И. Мальцев [53]). Для любьх натуральных чисел $k$ u $l$, больших 2, следующие условия әквивалентны:

(1) $G L(k, \mathbb{Z}) \equiv G L(l, \mathbb{Z})$

(2) $S L(k, \mathbb{Z}) \equiv S L(l, \mathbb{Z})$;

(3) $k=l$.

Здесь $\mathbb{Z}-$ кольио иельх чисел.

ТеОРема 1.1.3 (А.И. Мальцев [53]). Пусть F - поле нулевой характеристики $u k$ - любое натуральное число. Тогда следующие условия әквивалентнь:

(1) әлементарная теория $\mathscr{E} G L(k, F)$ разрешима $(k \geqslant 2)$;

(2) әлементарная теория $\mathscr{E} S L(k, F)$ разрешима $(k \geqslant 3)$;

(3) элементарная теория E्EF разрешима.

Теорема 1.1.4 (А.И. Мальцев [53]). Для любого натурального $k>2$ әлементарнье теории $\mathscr{E} G L(k, \mathbb{Z})$ и $\mathscr{E} S L(k, \mathbb{Z})$ неразрешимы.

Таким образом, уже в этой первой работе А.И. Мальцевым были поставлены и полностью решены проблемы классификации полей характеристики нуль, векторных пространств над этими полями и модулей над кольцом целых чисел с помошюю теорий производных структур - матричных групп в элементарном языке (единственном языке иерархии $E)$, а также вопросы разрешимости элементарных теорий этих матричных групп.

Результаты А.И. Мальцева получили дальнейшее развитие в работах [25], [27], [28], [8], где получены соответствуюшие утверждения для матричных груп и матричных колец над телами.

Теорема 1.1 .5 (К.И. Бейдар, А. В. Михалев [8]). Для произвольных тел $C, D$ и любых натуральных чисел $k, l$, больших 2 , следующие условия әквивалентнь:

(1) $G L(k, C) \equiv G L(l, D)$;

(2) $k=l$ и либо $C \equiv D$, либо $C \equiv D^{*}$.

Теорема 1.1.6 (В.Г. Дурнев [27]). Для любого тела $C$ и любого натурального числа $k$, большего двух, следующие условия әквивалентны:

(1) элементарная теория $\mathscr{E} G L(k, C)$ разрешима;

(2) әлементарная теория $\mathscr{E} C$ разрешима.

Теорема 1.1.7 (К.И. Бейдар, А.В. Михалев [8]). Пусть K, S- первичные кольча, содержащие $1\left(\frac{1}{2}\right), u k, l$ - натуральные числа, большие трех (двух). Тогда следуюшие условия эквивалентны:

(1) $G L(k, R) \equiv G L(l, S)$;

(2) $M(k, R) \equiv M(l, S)$ либо $M(l, R) \equiv M(k, S)^{*}$.

Таким образом, для полей, тел и ряда колец была получена достаточно исчерпывающая информация о проблемах классификации и разрешимости теорий производных структур матричных групп в языке тривиальной иерархии $E$ - в элементарном языке $\mathscr{E}$. 
Среди открытых и естественно возникающих в данном контексте вопросов отметим вопрос полного описания ассоциативных колец $R$, для которых условия "разрешима теория $\mathscr{E} R$ ” и “разрешима теория $\mathscr{E} G L(k, R)$ ” эквивалентны при $k>2$.

В связи с тем, что ряд результатов о равносильности элементарной эквивалентности колец $R$ и $S$ и элементарной эквивалентности групп $G L(k, R)$ и $G L(k, S)$ доказывался путем интерпретации кольца $R$ в групе $G L(k, R)$, представляет также интерес полное описание класса ассоциативных колец $R$, которые допускают элементарную интерпретацию в групе $G L(K, R)$ при $k>2$.

$\mathrm{K}$ настоящему времени накопилось достаточно много результатов, связанных с матричньми группами надцелыми числами для иных языков рассматриваемых иерархий.

Теорема 1.1.8 (Ю.В. Нагребецкая [65]). Для любых натуральньх чисел, больших чем два, следуюшие условия әквивалентны:

(1) $\forall \theta G L(k, \mathbb{Z})=\forall \theta G L(l, \mathbb{Z})$;

(2) $\forall \theta S L(k, \mathbb{Z})=\forall \theta S L(l, \mathbb{Z})$;

(3) $k=l$.

ТеОрема 1.1 .9 (А.М. Слободской [103]). Для любъх натуральных чисел $k$ u $l$ имеет место равенство

$$
\exists \forall \wedge \vee G L(k, \mathbb{Z})=\exists \forall \wedge \vee G L(l, \mathbb{Z})
$$

Теорема 1.1.10 (А.М. Слободской [103]). Теория $\forall \neg \vee G L(3, \mathbb{Z})$ неразрешима.

Теорема 1.1 .11 (В.Г. Дурнев [28]). Теории $\forall \exists \theta S L(k, \mathbb{Z})$ неразрешимы для лю6 бого $k>2$.

Теорема 1.1.12 (Ю.В. Нагребецкая [67]). Теории

$$
\exists \forall \wedge \vee G L(k, \mathbb{Z}) \quad u \quad \exists \forall \wedge \vee S L(k, \mathbb{Z})
$$

разрешимы для любого натурального $k$.

В работах В.Г. Дурнева [28], Ю. М. Важенина и Ю. В. Нагребецкой [126] доказан еще большой ряд утверждений, связанных с теориями груп $G L(k, \mathbb{Z})$ и $S L(k, \mathbb{Z})$ в различных элементарных языках.

Приведенные результаты позволяют ставить вопрос о нахождении границ разрешимости для групп $G L(k, \mathbb{Z}), S L(k, \mathbb{Z})$ в иерархии $S A$.

В работе Ю.В. Нагребецкой [66] доказано, что для любых $k \geqslant 2, l>2$ имеет место: $\exists \forall \wedge \vee S L(k, \mathbb{Z})=\exists \forall \wedge \vee S L(l, \mathbb{Z}), \forall \exists S L(2, \mathbb{Z}) \neq \forall \exists S L(l, \mathbb{Z}), \exists \forall \neg S L(2, \mathbb{Z}) \neq$ $\exists \forall \neg S L(l, \mathbb{Z})$. Наряду с приведенными вьше результатами это влечет следующее утверждение.

Теорема 1.1.13 (Ю.В. Нагребецкая [66]). SA-границей различимости для класса $\{S L(k, \mathbb{Z}) \mid k \geqslant 2\}$ является множество $\{\forall \exists, \exists \forall \neg, \forall \neg \vee, \exists \neg \wedge\}$.

Ряд результатов по вопросам разрешимости элементарных теорий групп унитреугольных матриц и вопросам об элементарной эквивалентности этих груп был получен О. В. Белеградеком в работах [10]-[18]. Заметим, что группа $U T(2, R)$ изоморфна аддитивной групше кольца $R$. 
Теорема 1.1.14 (О.В. Белеградек [18]). Для любых колеи $R$ и $S$, хотя бы одно из которых коммутативно или без делителей нуля, и любых натуральных чисел $k$ и l, больших двух, следующие условия әквивалентны:

(1) $U T(k, R) \equiv U T(l, S)$;

(2) $k=l$ и либо $R \equiv S$, либо $R \equiv S^{*}$.

Теорема 1.1.15 (О.В. Белеградек [18]). Для любого коммутативного кольца, либо кольца без делителей нуля $R$ и любого натурального $k>2$ следующие условия эквивалентны:

(1) әлементарная теория $\mathscr{E} U T(k, R)$ разрешима;

(2) әлементарная теория $\mathscr{E} R$ разрешима.

Существенность условий на кольцо $R$ в условиях теорем 1.1.14, 1.1.15 демонстрируют следующие результаты.

Теорема 1.1 .16 (О.В. Белеградек [18]). Существуют конечные кольца $R$ и $S$ такие, что для любого натурального $k \geqslant 2$ имеет место

$$
U T(k, R) \cong U T(k, S), \quad \text { но } \quad R \nsubseteq S \quad \text { u } \quad R \nsubseteq S^{*}
$$

Теорема 1.1.17 (О.В. Белеградек [18]). Существует кольио $R$ такое, что его элементарная теория $\mathscr{E} R$ неразрешима, а теория $\mathscr{E} U T(k, R)$ разрешима для любого натурального $k>2$.

В работах О.В. Белеградека рассмотрен целый ряд иных вопросов, связанных с элементарными теориями групп вида $U T(k, R)$. В частности, описаны все групшы, элементарно эквивалентные группам вида $U T(k, R)$.

Известен ряд результатов, связанных с теориями унитреугольных матричных групा в иных, отличных от элементарного, языках.

Заметим лишш, что из результатов А. И. Мальцева [52] и Б. Роуза [89] очевидньм образом вытекает неразрешимость сколемовской теории $\forall \exists \theta U T(k, \mathbb{Z})$ при $k>2$.

Перейдем теперь к изложению результатов о матричных кольцах.

Элементарная классификация колец $M(k, R)$ для случая тел $R$ была получена еше А.И. Мальцевым.

Теорема 1.1.18 (А. И. Мальцев [52]). Для любьх тел D, C и натуральных чисел $k$ и l следующие условия эквивалентнь:

(1) $M(k, D) \equiv M(l, C)$;

(2) $k=l$ и либо $D \equiv C$, либо $D \equiv C^{*}$.

Аналогичный результат имеет место и для ряда ассоциативных колец.

Теорема 1.1.19 (Ю.В. Нагребецкая). Пусть $R$ и $S$ - ассоииативные коммутативные кольца с единицей и без делителей нуля. Тогда для любых натуральных $k$ u l следующие условия әквивалентны:

(1) $M(k, R) \equiv M(l, S)$;

(2) $R \equiv S u k=l$.

Этот результат следует из интерпретации ассоциативного коммутативного кольца $R$ с единицей и без делителей нуля при помоши бескванторной формулы, приведенной в $[65]$, в кольце $M(k, R)$. 
Ю. В. Нагребецкой [65] доказано также, что если $R$ ассоциативное коммутативное кольцо, то разрешимость теории $\mathscr{E} R$ эквивалентна разрешимости теории $\mathscr{E} M(k, R)$.

Для более узкого класса колец имеет место следующий более сильньй результат, полученный на основе результатов Ж. Дьёдонне [29].

Теорема 1.1.20 (Ю.В. Нагребецкая). Если $R$ - ассоциативное коммутативное кольио с единицей и без делителей нуля или тело нулевой либо нечетной характеристики, то для любого натурального $k$ имеет место совпадение грании, разрешимости в схемной иерархии:

$$
B_{S}(M(k, R)) \equiv B_{S}(R) .
$$

Это утверждение легко следует из определимости подобного кольца $R$ в кольце $M(k, R)$ с помошью формулы $\varphi(x)=\forall y(x y=y x)$.

Полное описание гранищ разрешимости матричных колец над кольцом $\mathbb{Z}$ в схемно-альтернативной иерархии содержится в следующем утверждении.

ТЕОРема 1.1.21 (Ю.В. Нагребецкая [65]). Для любого натурального числа $k$ $S A$-граница разрешимости кольца $M(k, \mathbb{Z})$ определяется равенством

$$
B_{S A}(M(k, \mathbb{Z}))=\{\forall \exists, \exists \forall, \forall \neg \vee, \exists \neg \wedge\} .
$$

Изучение элементарных теорий колец $N T(k, R)$ было начато Б. Роузом [89], В. Вилером [129] и продолжено К.Р. Виделой [128].

Теорема 1.1 .22 (К.Р. Видела [128]). Для любых колеи, $R$ и $S$ и любых натуральных чисел $k$ и $l$ следующие условия әквивалентны:

(1) $N T(k, R) \equiv N T(l, S)$;

(2) $k=l$ и либо $R \equiv S$, либо $R \equiv S^{*}$.

Теорема 1.1.23 (К.Р. Видела [128]). Для произвольного кольца $R$ и любого натурального числа $k$ следующие условия эквивалентны:

(1) элементарная теория $\mathscr{E} N T(k, R)$ разрешима;

(2) элементарная теория $\mathscr{E} R$ разрешима

Заметим, что так как группы $G L(k, R), S L(k, R), U T(k, R)$ и кольца $M(k, R)$, $N T(k, R)$, очевидным образом, интерпретируются с помощью конъюнкций атомарных формул в самом кольце $R$, то для доказательства большей части приведенных в данном разделе 1.1 результатов достаточно построить интерпретацию, в соответствующем языке, кольца $R$ в рассматриваемой матричной группе или кольце.

Другой метод нахождения критериев элементарной эквивалентности матричных групп и колец над кольцами $R$ и $S$ основан на известной теореме Кейслера-Шелаха (см. [94]) об изоморфизме подходяших ультрастепеней элементарно эквивалентных алгебраических систем, на том, что ультрастепень матричной группы (кольца) над кольцом $R$ изоморфна матричной групе (кольцу) над соответствуюшей ультрастепенњю кольца $R$, и на описании абстрактных изоморфизмов матричных групп (колец). (См., к примеру, [35], [36].)

Отметим также, что цельй ряд иных, близких к приведенньм вьше результатов о теориях матричных групп и колец в языках первого порядка приведены в работах К. И. Бейдара, А. В. Михалева и Г.Е. Пунинского [9], О. В. Белеградека [10]-[18], Ю. В. Нагребецкой [63]-[67], Е.И. Буниной [21]-[25], В.Г. Дурнева [27], [28], В. Н. Ремесленникова и В. А. Романькова [88], В. А. Толстых [109] и др. 
1.2. Полугруппы эндоморфизмов и группы автоморфизмов бесконечномерных векторных пространств. В настоящем разделе рассмотрены вопросы элементарной классификации и разрешимости элементарных теорий полугрупп и групп линейных преобразований бесконечномерных, в отличие от раздела 1.1, векторных пространств. Довольно естественно, что в ответах на эти вопросы играет роль логика второго порядка, т.е. расширение логики первого порядка, в котором допускается навешивание кванторов $\forall$ и $\exists$ по произвольным предикатам, определенным на основном множестве рассматриваемой алгебраической системы. Через $\mathrm{Th}_{2}(\mathfrak{A})$ обозначим далее теорию алгебраической системы $\mathfrak{A}$ в логике второго порядка, а через $\operatorname{Th}_{2}^{k}(\mathfrak{A})$, где $k$-некоторый кардинал, теорию алгебраической системы $\mathfrak{A}$ в расширении логики первого порядка, допускаюшем навешивание кванторов $\forall$ и $\exists$ по произвольным предикатам мощности, не превышающей кардинал $k$.

Для любого тела $D$ и кардинала $k$ пусть $V_{k}^{D}$ - векторное пространство над телом $D$ размерности $k, \operatorname{End}\left(V_{k}^{D}\right)\left(\operatorname{Aut}\left(V_{k}^{D}\right)\right)$ - полугруппа (группа) всех эндоморфизмов (автоморфизмов) векторного пространства $V_{k}^{D}$, a $\operatorname{End}^{*}\left(V_{k}^{D}\right)$ - кольцо всех эндоморфизмов этого векторного пространства. Через $\langle k, D\rangle$ обозначим двусортную алгебраическую систему, первьй сорт которой - множество $k$ без каких-либо отношений и функций, а второй - тело $D$ в стандартной сигнатуре.

Пусть $K$ обозначает класс тел $D$, удовлетворяющих условию: мошность числа классов сопряженных элементов мультипликативной группы $D^{*}$ равна мощности групшы $D^{*}$. Заметим, что $K$ - довольно широкий класс тел, включающий в себя, в частности, все поля.

Имеет место следуюший результат, даюший исчерпывающий ответ об элементарной эквивалентности полугрупп (колец) эндоморфизмов и груп автоморфизмов бесконечномерных векторных пространств над телами.

Теорема 1.2.1 (В.А. Толстых [111]). Для любъх тел D, S из класса $K$ и любых бесконечных кардиналов $k$ и $\lambda$ следуюшие условия эквивалентны:

(1) $\operatorname{End}^{*}\left(V_{k}^{D}\right) \equiv \operatorname{End}^{*}\left(V_{\lambda}^{S}\right)$

(2) $\operatorname{End}\left(V_{k}^{D}\right) \equiv \operatorname{End}\left(V_{\lambda}^{S}\right)$;

(3) $\operatorname{Aut}\left(V_{k}^{D}\right) \equiv \operatorname{Aut}\left(V_{\lambda}^{S}\right)$;

(4) $\operatorname{Th}_{2}^{k}(\langle k, D\rangle)=\operatorname{Th}_{2}^{\lambda}(\langle\lambda, S\rangle)$.

Как замечено в [111], равенство $\operatorname{Th}_{2}^{k}(\langle k, D\rangle)=\operatorname{Th}_{2}^{\lambda}(\langle\lambda, S\rangle)$ влечет равенства $\operatorname{Th}_{2}(k)=\operatorname{Th}_{2}(\lambda)$ и $\operatorname{Th}_{2}^{k}(D)=\operatorname{Th}_{2}^{\lambda}(S)$ (но не сводится к ним). Заметим также, что если в качестве $D$ выбрать любое тело из класса $K$, описьваемое с точностью до изоморфизма одним предложением в логике второго порядка, то при $k \geqslant|D|$ равенство $\operatorname{Th}_{2}^{k}(\langle k, D\rangle)=\operatorname{Th}_{2}^{\lambda}(\langle\lambda, S\rangle)$ равносильно тому, что $\lambda \geqslant|S|, \operatorname{Th}_{2}(k)=\operatorname{Th}_{2}(\lambda)$ и $D \cong S$. В качестве тел $D$ указанного вида могут фигурировать поля $\mathbb{Q}, \mathbb{R}$ и $\mathbb{C}$, счетные алгебраически замкнутые и конечные поля.

Поскольку доказательство теоремы 1.2.1 состоит в построении биинтерпретации элементарных теорий группы $\operatorname{Aut}\left(V_{k}^{D}\right)$ и теории второго порядка $\operatorname{Th}_{2}^{k}(\langle k, D\rangle)$ друг в друге, то имеет место и следующее утверждение.

ТеОрема 1.2.2 (В.А. Толстых [111]). Для любого бесконечного кардинала $k u$ любого тела $D$ из класса $K$ әлементарнье теории группь $\operatorname{Aut}\left(V_{k}^{D}\right)$, полугруппь $\operatorname{End}\left(V_{k}^{D}\right)$ икольиа $E^{*}\left(V_{k}^{D}\right)$ неразрешимы.

В работе [111] содержится еше целый ряд утверждений, связанных с элементарными теориями различных групп и полугрупп преобразований бесконечномерных век- 
торных пространств. Близкие вопросы рассматриваются в работе [127].

В связи с утверждением теоремы 1.2.2 естественным образом возникает вопрос о нахождении границ разрешимости теорий первого порядка указанных в этой теореме груп, полугрупп и колец в различных иерархиях язьков первого порядка.

Кроме того, остается открытым вопрос о справедливости утверждений теорем 1.2.1 и 1.2.2 для произвольных тел. Заметим лишь, что, как указано в работе [111], утверждения теорем 1.2.1 и 1.2.2 имеют место для произвольных тел $D, S$ в случае, когда размерности $k, \lambda$ векторных пространств удовлетворяют неравенствам $k, \lambda>$ $\max \{|D|,|S|\}$.

\section{§. Производные структуры полугрупп и групп}

2.1. Решетки подполугрупп и подгрупп. Класс $\mathfrak{K}$ полугрупп назьвается решеточно конечно аксиоматизируемылм, если класс решеток $\mathrm{Sub} \mathfrak{K}=\{\mathrm{Sub} \mathfrak{A} \mid$ $\mathfrak{A} \in \mathfrak{K}\}$ аксиоматизируем внутри класса Sub $\mathfrak{C}$, где $\mathfrak{C}-$ класс всех полугруп, т.е. если существует такой конечно аксиоматизируемый класс решеток $\mathscr{R}$, что Sub $\mathfrak{K}=\mathscr{R} \cap \operatorname{SubC}$. Полугруппы $S$ и $T$ назьваются решеточно әлементарно әквивалентными, если $\operatorname{Sub} S \equiv \operatorname{Sub} T$. Говорят, что класс полугруппы $\mathfrak{K}$ решеточно элементарно замкнут, если класс решеток Sub $\mathfrak{K}$ элементарно замкнут внутри класса Sub $\mathfrak{C}$, т.е. если для полугруп $S$ и $T$, где $S \in \mathfrak{K}$, отношение $\operatorname{Sub} S \equiv \operatorname{Sub} T$ влечет включение $T \in \mathfrak{K}$. Если при этом $\mathfrak{K}=I\{T\}$ и $\mathfrak{K}$ решеточно элементарно замкнут, то полугрупу $T$ будем назьвать решеточно элементарно определимой. Здесь $I \mathfrak{K}=\{\mathfrak{A} \mid$ сушествует $\mathfrak{B} \in \mathfrak{K}$ такая, что $\mathfrak{A} \cong \mathfrak{B}\}$.

Л. Н. Шевриньм получен цельй ряд результатов о решеточной элементарной определимости и аксиоматизируемости различных полугрупп и их классов.

Теорема 2.1.1 (Л.Н. Шеврин [100], [101]). Решеточно конечно аксиоматизируемы классы полугрупп идемпотентов, полугрупп без идемпотентов, периодических полугрупп, периодических унипотентных полугрупп, непериодических полугрупп, квазичиклических групп, групп без кручения, непериодических групп, непериодических абелевых групп, линейно упорядочиваемых групп.

Теорема 2.1.2 (Л.Н. Шеврин [100], [101]). Решеточно аксиоматизируемы классы коммутативных полугрупп с законом сокращения и без идемпотентов, свободных полугрупп, свободных коммутативных полугрупп, свободных полугрупп идемпотентов.

ТЕОРема 2.1.3 (Л. Н. Шеврин [100], [101]). Бесконечная ииклическая полугруппа и бесконечная ииклическая группа решеточно әлементарно определимы.

Довольно полная информация по вопросам разрешимости элементарных теорий решеток подполугрупп содержится в следуюших результатах.

ТеОРема 2.1.4 (Ю.М. Важенин [119]). Если класс $\mathfrak{K}$ полугрупп содержит непериодическую полугруппу, либо индексы конечных ииклических подполугрупп полугрупп из $\mathfrak{K}$ не ограничены в совокупности, то элементарная теория классов решеток Sub $\mathfrak{K}$ наследственно неразрешима.

Теорема 2.1.5 (Ю. М. Важенин, Е. В. Линецкий [125]). Наследственно неразрешима теория класса $\operatorname{Sub} \mathfrak{K}$, где $\mathfrak{K}$ - класс всех конечных полурешеток. 
Полностью решена проблема разрешимости элементарной теории класcа Sub $\mathfrak{K}$ в случае, когда $\mathfrak{K}$ - некоторое многообразие полугруп.

Теорема 2.1.6 (Б. Баясгалан [6]). Для любого многообразия $\mathfrak{M}$ nолугрупп следующие условия әквивалентны:

(1) әлементарная теория класса Sub $\mathfrak{M}$ разрешима;

(2) либо $\mathfrak{M} \subset \operatorname{Var}\{x y z=x z\}, л u б o \mathfrak{M} \subseteq \operatorname{Var}\{x y z=x\} \times \operatorname{Var}\left\{x^{n} y=y, x y=y x\right\}$ для некоторого $n \geqslant 1$.

Здесь $\operatorname{Var}\left\{t_{1}=t_{2}\right\}$-многообразие, аксиоматизируемое тождеством $t_{1}=t_{2}$.

Представляет интерес нахождения $S A$-критических теорий для класса Sub $\mathfrak{M}$, где $\mathfrak{M}$ - многообразие всех полугрупп.

Изучение элементарных теорий решеток подгрупп различных групп было начато в работе М.И. Каргаполова [45]. Дальнейшие результаты по разрешимости подобных элементарных теорий были получены в работах Г. Т. Козлова [46], Н. Г. Луковникова и В. И. Мартьянова [51], Э. И. Фридмана [34] и М. А. Тайцлина [107]. Из результатов работы М. И. Каргаполова и теоремы 2.1.6 вытекает следуюшее утверждение.

Теорема 2.1.7 (Б. Баясгалан [5]). Для любого многообразия М̆ групп следующие условия әквивалентны:

(1) элементарная теория класса Sub M, pешеток подгрупn $\mathfrak{M - z p y n n , ~ р а з р е - ~}$ шима;

(2) М-многообразие абелевьх периодических групп.

Известен также ряд результатов о неразрешимости универсальных теорий решеток подгрупп различных классов групп. Из доказанной в работе [50] неразрешимости квазиэквациональной теории класса всех (всех конечных) модулярных арговых решеток и известной вложимости этих решеток в решетки подгрупп абелевых групп вытекает следующее утверждение.

ТЕОРема 2.1.8 (Л. Липшиц [50]). Неразрешима универсальная теория решеток подгрупп всех (всех конечных) абелевых групп.

Имеет место также следующая теорема.

Теорема 2.1.9 (А. М. Слободской, Э.И. Фридман [104]). Неразрешимы универсальные теории решеток подгрупп (сервантных подгрупп) следующих классов абелевых групп: всех, всех без кручения, всех периодических, всех р-групп для некоторого простого р, всех полных, всех полных без кручения.

Проблема классификации абелевых груп по элементарным теориям решеток их подгрупп решена А.И. Кокориным.

Теорема 2.1.10 (А.И. Кокорин [47]). Для любых абелевых групn $G$ u $G^{\prime}$ элементарная әквивалентность $\mathrm{Sub} G \equiv \operatorname{Sub} G^{\prime}$ ux решеток подгрупп влечет их разложимость $G \cong H \oplus Q, G^{\prime} \cong H^{\prime} \oplus Q^{\prime}$ такую, ито $H \equiv H^{\prime}$, а группьи $Q$ и $Q^{\prime}$ локально ииклические.

СлЕДСТВИЕ 2.1.1 (А.И. Кокорин [47]). Для любых абелевьх групп $G$ u $G^{\prime}$ без кручения ранга, не меньшего двух, әлементарная әквивалентность $\mathrm{Sub} G \equiv$ 
Sub $G^{\prime}$ их решеток подгрупп влечет әлементарную әквивалентность $G \equiv G^{\prime}$ самих әрynn.

В связи с приведенными результатами представляет интерес вопрос нахождения гранищ разрешимости в иерархиях $V, S, A, V A, S A$ класса Sub $\mathfrak{K}$, где $\mathfrak{K}$ - класс всех полугруп, класс всех груп, класс всех абелевых групп.

Заметим в заключение, что еше одной производной структурой, связанной с решетками подалгебр и вопросами порожденности подалгебр, могут служить так называемые генерические мультиалгебры (см. [77], [83]).

2.2. Решетки конгруэнций и близкие к ним производные структуры. Через Id $S$ и $\operatorname{Div} S$ обозначим соответственно решетку идеалов полугрупшы $S$ и квазиупорядоченное множество $\langle S ; \mid\rangle$, где |- отношение делимости, определенное на основном множестве полугруппы $S$ следуюшим образом:

$$
x \mid y \Leftrightarrow \exists a, b \in S(y=a x b \vee y=a x \vee y=x b \vee x=y) .
$$

Эти производные структуры связаны между собой и с решеткой конгруэнций Con $S$ полугрупшы $S$. А именно: решетка Id $S$ является полной дистрибутивной подрешеткой решетки Con $S$ и антиизоморфна решетке $2^{\operatorname{Div} S}$ - кардинальной степени двухэлементной решетки 2 с показателем - квазиупорядоченным множеством Div $S$.

Как и ранее, для класса $\mathfrak{K}$ полугрупп через $\operatorname{Con} \mathfrak{K}, \operatorname{Id} \mathfrak{K}, \operatorname{Div} \mathfrak{K}$ обозначим соответственно классы $\{\operatorname{Con} S \mid S \in \mathfrak{K}\},\{\operatorname{Id} S \mid S \in \mathfrak{K}\},\{\operatorname{Div} S \mid S \in \mathfrak{K}\}$.

Имеется довольно полная информация по вопросу разрешимости элементарных теорий классов Con $\mathfrak{M}, \operatorname{Id} \mathfrak{M}, \operatorname{Div} \mathfrak{M}$ для различных многообразий $\mathfrak{M}$ полугрупп.

Теорема 2.2.1 (Б. Баясгалан [3]). Класс решеток Con $\mathfrak{M}$ имеет разрешимую әлементарную теорию только для многообразий $\mathfrak{M}$ полугрупп вида $\mathfrak{M}=$ $\operatorname{Var}\left\{x^{n} y=y, x y=y x\right\}$ для некоторого натурального $n$.

СлЕДСТВИЕ 2.2.1 (Б. Баясгалан [3]). Для многообразий М групn следующие условия әквивалентнь:

(1) разрешима әлементарная теория класса решеток Con $\mathfrak{M}$ (т.е. решеток нормальных подгрупп М-групп);

(2) разрешима әлементарная теория класса решеток Sub M (решеток подzpynn $\mathfrak{M - z p y n n ) . ~}$

Теорема 2.2.2 (Б. Баясгалан [3]). Для многообразия М полугрупn следующие условия эквивалентнь:

(1) разрешима әлементарная теория класса квазиупорядоченных множеств $\operatorname{Div} \mathfrak{M}$

(2) разрешима әлементарная теория класса решеток Id $\mathfrak{M}$;

(3) многообразие $\mathfrak{M}$ не включает в себя многообразия вида $\operatorname{Var}\left\{x y z=t^{2}\right.$, $x y=y x\} u \operatorname{Var}\left\{x^{2}=x, x y=y x\right\}$

(4) на многообразии $\mathfrak{M}$ выполнено некоторое тождество вида ихv $=y z$, где $x, y, z$ - переменные, а $u, v$ - некоторые полугрупповые термы;

(5) каждая полугруппа из М является идеальным расширением вполне простой полугруппь при помощи полугруппь с нулевым умножением.

В некоторых случаях найдены границы разрешимости рассматриваемых выше теорий. K примеру, имеет место следуюшая теорема. 
Теорема 2.2.3 (Б. Баясгалан [4]). Если $\mathfrak{K}$ - произвольный класс полугрупn, содержащий все инверсные полугруппь, либо $\mathfrak{K}$ - конечно базируемое многообразие локально конечных полугрупп, включающее в себя многообразие полурешеток, то $S A$-граница разрешимости класса $\operatorname{Div} \mathfrak{K}$ есть $\{\exists \forall \neg \wedge \vee\}$.

Теорема 2.2 .4 (Б. Баясгалан [5]). Для произвольного многообразия М полугрупп следующие условия эквивалентны:

(1) әлементарная теория класса Id $\mathfrak{M}$ неразрешима;

(2) SA-граница разрешимости класса Id $\mathfrak{M}$ есть $\{\exists \forall \exists \neg \wedge \vee\}$;

(3) $\operatorname{Var}\left\{x^{2}=x, x y=y x\right\} \subseteq \mathfrak{M}$ uлu $\operatorname{Var}\left\{x y z=t^{2}, x y=y x\right\} \subseteq \mathfrak{M}$.

2.3. Степени полугрупп. Для полугруппы $\mathfrak{C}=\langle S ; \cdot\rangle$ через Pow $\mathfrak{C}$ обозначим множество всех непустых подмножеств множества $S$ с операцией умножения комплек-

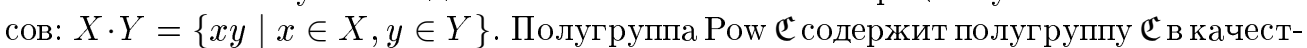
ве своей подполугруппы, если допустить отождествление элементов из $S$ с соответствующими одноэлементньми подмножествами. Отметим, что в работе Е. М. Могилянской [60] построен пример двух неизморфных полугрупп $\mathfrak{C}_{1}$ и $\mathfrak{C}_{2}$, степени которых, полугрупшы Pow $\mathfrak{C}_{1}$ и Pow $\mathfrak{C}_{2}$, изоморфны.

Для полных симметрических полугрупп (полугрупп всех отображений множества в себя) имеет место следуюшие утверждение.

Теорема 2.3.1 (Ю.М. Важенин [117]). Полная симметрическая полугруппа сильно элементарно определима своей степенью, более того, отношения Pow $\mathfrak{C}_{1} \equiv$ Pow $\mathfrak{C}_{2} \quad$ u $\mathfrak{C}_{1} \equiv \mathfrak{C}_{2}$ равносильны для любых полных симметрических nолугрупп $\mathfrak{C}_{i}$.

Следуюшая теорема дает описание всех полугрупповых многообразий $\mathfrak{M}$, для которых соответствующий класс Pow $\mathfrak{M}=\{$ Pow $\mathfrak{C} \mid \mathfrak{C} \in \mathfrak{M}\}$ имеет разрешимую элементарную теорию.

Теорема 2.3.2 (Б. Баясгалан [3]). Для многообразия $\mathfrak{M}$ полугрупn класс Pоw $\mathfrak{M}$ имеет разрешимую әлементарную теорию тогда и только тогда, когда $\mathfrak{M} \subset$ $\operatorname{Var}\{x y z=x z\}$. Отсюда, в частности, следует, что для любого нетривиального группового многообразия $\mathfrak{M}$ класс Pow $\mathfrak{M}$ имеет неразрешимую әлементарную теорию.

Теорема 2.3.3 (Б. Баясгалан, Ю. М. Важенин [7]). SA-граница разрешимости класса степеней всех полугрупп есть $\{\forall \neg \vee\}$.

\section{§ 3. Преобразования упорядоченных множеств и графов}

\section{1. Полугруппы эндоморфизмов и направленных преобразований.}

Пусть $\mathfrak{G}=\langle\Gamma ; \alpha\rangle-$ произвольньй граф, т.е. непустое множество вершин с определенным на нем бинарным рефлексивным отношением $\alpha$. Граф̆ $\mathfrak{G}$ назовем пустьм (тривиальньм $)$, если $\alpha$ есть отношение равенства на $\Gamma(\alpha=\Gamma \times \Gamma)$. Через $\mathfrak{G}^{-1}$ обозначим графф $\left\langle\Gamma ; \alpha^{-1}\right\rangle$, а через $E(\mathfrak{G}), I(\mathfrak{G}), D(\mathfrak{G})$ - полугрупшы всех эндоморфизмов, inf-эндоморфизмов и частичный группоид направленных преобразований графа $\mathfrak{G}$ соответственно. При этом для любого класса $\mathfrak{K}$ граффов положим $E(\mathfrak{K})=\{E(\mathfrak{G}) \mid \mathfrak{G} \in \mathfrak{K}\}$, аналогично определяются $I(\mathfrak{K})$ и $D(\mathfrak{K})$. Напомним, что

$$
\begin{aligned}
f & \in E(\mathfrak{G}) \Leftrightarrow \forall x, y \in \Gamma(\alpha(x, y) \rightarrow \alpha(f(x), f(y))), \\
f \in I(\mathfrak{G}) & \Leftrightarrow \forall x, y, z \in \Gamma(x \in \inf (y, z) \rightarrow f(x) \in \inf (f(y), f(z))),
\end{aligned}
$$


где $\inf (y, z)=\{x \in \Gamma \mid t(\alpha)(x, y) \& t(\alpha)(x, z) \wedge \forall v(t(\alpha)(v, y) \& t(\alpha)(v, z) \rightarrow t(\alpha)(v, x))\}$, a $t(\alpha)$ - транзитивное замькание отношения $\alpha$.

Наконец, $f \in D(\mathfrak{G})$, если $\forall x \in \Gamma \alpha(x, f(x))$.

Пусть $X \in\{E, I, D\}$. Класс графов $\mathfrak{K}$ назовем (конечно) $X$-аксиоматизируемьлм, если класс $X(\mathfrak{K})$ полугрупп, или, соответственно, частичных группоидов, относительно (конечно) аксиоматизируем внутри класса $X(\mathfrak{G} r)$, где $\mathfrak{G} r$ - класс всех графов. Класс графов $\mathfrak{K}\left(\right.$ точно) элементарно $X$-определим, если для любого графа $\mathfrak{G}_{2}$ из соотношения $X\left(\mathfrak{G}_{1}\right) \equiv X\left(\mathfrak{G}_{2}\right)$ для некоторого графа $\mathfrak{G}_{1} \in \mathfrak{K}$ следует, что $\mathfrak{G}_{1} \equiv \mathfrak{G}_{2}$ или $\mathfrak{G}_{1}^{-1} \equiv \mathfrak{G}_{2}$ (соответственно $\left.\mathfrak{G}_{1} \equiv \mathfrak{G}_{2}\right)$.

Имеют место следуюшие утверждения, связанные с $X$-аксиоматизируемостью и элементарной $X$-определимостью различных классов графов.

Теорема 3.1.1 (Ю.М. Важенин [115], [116]). Следуюшие классы графов являются конечно $X$-аксиоматизируемыми для $X \in\{E, I\}:$ класс $\mathfrak{A}_{1}$ всех симметричных графов; класс $\mathfrak{A}_{2}$ всех графов, в каждом из которых есть дуга, не принадлежащая ни одному ориентированному циклу; класс $\mathfrak{A}_{3}$ всех тривиальных графов; класс всех антисимметричных графов; класс всех транзитивных графов; класс всех линейно упорядоченных множеств.

ТеОРема 3.1.2 (Ю.М. Важенин [115], [116]). Произвольный граф из класса $\left(\mathfrak{A}_{1} \cup \mathfrak{A}_{2}\right) \backslash \mathfrak{A}_{3}$ (в частности, любое частично упорядоченное мнохество) является әлементарно $X$-определимым для $X \in\{E, I\}$.

Теорема 3.1.3 (Ю.М. Важенин [115], [116]). Для $X \in\{E, I\}$ класс полугрупn $X(\mathfrak{K})$ арифметически не замкнут и, следовательно, не является $X$-аксиоматизируемьм, если класс графов $\mathfrak{K}$ содержит бесконечный граф одного из следующих типов: неантисимметричный, пустой; линейно упорядоченное множество; решетка с нулем и единицей, все остальные әлементы которой попарно несравнимьи (решетка вида $M_{k}$ для произвольного кардинала $k$ ).

Точную (верхнюю или нижнюю) грань элементов $x, y$ некоторого частично упорядоченного множества назовем нетривиальной, если $x$ и $y$ несравнимы.

ТЕорема 3.1 .4 (Ю.М. Важенин [116]). Любое частично упорядоченное множество, содержащее хотя бы одну нетривиальную точную грань, является точно әлементарно I-определимым.

Через $E_{w}(\mathfrak{G})\left(E_{n}(\mathfrak{G})\right)$ обозначим подполугруппу полугруппы $E(\mathfrak{G})$, состоящую из эндоморфизмов графа $\mathfrak{G}$ с произвольной конечной (состояшей не более чем из $n$ элементов) областью значений.

ТЕОРема 3.1 .5 (А.Г. Пинус [69]). Для любого класса $\mathfrak{K}$ графов, включающего в себя бесконечное линейно упорядоченное множество, элементарные теории классов полугрупп $E(\mathfrak{K}), E_{w}(\mathfrak{K})$ неразрешимы. Для класса $\mathfrak{K}$, состоящего из всех линейно упорядоченных множеств, и любого натурального $n$ әлементарная теория класса полугрупп $E_{n}(\mathfrak{K})$ разрешима.

Заметим также, что из результатов работы А. Г. Пинуса [70] следует, что $E_{n}-$ число Левенгейма для класса всех линейно упорядоченных множеств - равно $2^{\aleph_{0}}$.

В дальнейших утверждениях рефлексивность рассматриваемых графов не предполагается. 
Теорема 3.1.6 (Ю. М. Важенин [116], [120]). Следующие классы графов конечно D-аксиоматизируемы: класс всех транзитивных графов; класс всех пустых графов; класс всех непустых антисимметричных графов; класс всех симметричных графов; класс всех частично упорядоченных множеств, разложимых в кардинальную сумму множсеств с наибольшим и наименьшим әлементом, лишь одно из которьх неодноэлементно.

Теорема 3.1.7 (Ю.М. Важенин [116], [120]). Для произвольных графов $\mathfrak{G}_{1} u \mathfrak{G}_{2}$ отношение $D\left(\mathfrak{G}_{1}\right) \equiv D\left(\mathfrak{G}_{2}\right)$ влечет отношение $\mathfrak{G}_{1}^{\prime} \equiv \mathfrak{G}_{2}^{\prime}$, где граф $\mathfrak{G}^{\prime}$ получается из графа $\mathfrak{G}$ удалением изолированных вершин.

В работах Ю. М. Важенина [115], [116], [120] получен целый ряд утверждений- аналогов утверждений теорем 3.1.1-3.1.4, 3.1.6 и 3.1.7 для полугрупп и группоидов частичных преобразований граффов (см. также работы В. А. Молчанова [61] и Ю. М. Важенина [124]).

3.2. Симметрические полугруппы и группы. Особняком стоит вопрос о полугрупшах преобразований тривиальных графов $\mathfrak{G}$ вида $\langle A ; \alpha\rangle$, где $\alpha=A \times A$, т.е. о полных симметрических полугруппах $\operatorname{Syms} A$ на множествах $A$, а также о симметрических группах $\operatorname{Sym} A$. Здесь получены окончательные ответы для этих полугруп и групп на все основные рассматриваемые вьше вопросы.

Прежде всего заметим, что достаточно очевидным образом (см. [118] ) в элементарной теории полугруппы Syms $A$ интепретируется полная логика второго порядка на множестве $A$ (логика, допускаюшая навешивание кванторов $\forall$ и $\exists$ по произвольным предикатам на множестве $A$ ). Тем самым, отношение Syms $A \equiv \operatorname{Syms} B$ равносильно совпадению теорий множеств $A$ и $B$ в полной логике второго порядка (отношению $\left.A \equiv_{2} B\right)$.

Иная ситуация складьвается при рассмотрении симметрических груп $\operatorname{Sym} A$. Заметим, что при доказательстве неразрешимости элементарной теории класса всех конечных симметрических групп в работе Ю. Л. Ершова [30] была построена интерпретация двухосновной модели $\langle A ; \operatorname{Sym} A$, o $\rangle$ в симметрической групе $\operatorname{Sym} A$, здесь отношение о $(a, b, c)$ имеет место тогда и только тогда, когда $c \in \operatorname{Sym} A, a, b \in A$ и $c(a)=b$. На основе этого ряд частных результатов об элементарной определимости групп вида Sym $A$ в классе всех полных симметрических груп был получен в работах Р. Маккензи [59] и А. Г. Пинуса [71]. Окончательное решение этой, а вместе с тем и всех иных проблем, связанных с элементарными теориями симметрических групп, был дан в работе С. Шелаха [96].

Пусть $\Omega=\left(2^{\aleph_{0}}\right)^{+}$. Для любого ординала $\alpha$ сушествует единственное его представление в виде

$$
\alpha=\Omega^{w} \alpha_{w}+\cdots+\Omega^{n} \alpha_{[n]}+\cdots+\Omega \alpha_{[1]}+\alpha_{[n]},
$$

где $\alpha_{[i]}<\Omega$. Пусть

$$
\alpha[n]=\Omega^{w} \alpha_{w}+\cdots+\Omega^{n+1} \alpha_{[n+1]}, \quad \alpha^{[n]}= \begin{cases}1+c f \alpha[1], & \text { если } c f \alpha[n]<\Omega \\ 0 & \text { иначе. }\end{cases}
$$

Через $U_{\alpha}$ обозначим множество мошности $\min \left\{2^{\aleph_{0}}, \aleph_{\alpha}\right\}$, а через $M_{\alpha}$ - многоосновную модель $\left\langle U_{\alpha}, \alpha_{[0]}, \alpha_{[1]}, \ldots, \alpha^{[0]}, \alpha^{[1]}, \ldots ;<\right\rangle$, где множества $U_{\alpha}, \alpha_{[i]}, \alpha^{[j]}$ предполагаются дизъюнктными, а предикат < интепретируется как обычньй порядок на ординалах $\alpha_{[i]}, \alpha^{[j]}$ (т.е. на совокупностях меньших ординалов). 
Теорема 3.2 .1 (С. Шелах [96]). Для любых кардиналов $\aleph_{\alpha} u \aleph_{\beta}$ следующие условия әквивалентнь:

a) $\operatorname{Sym}\left(\aleph_{\alpha}\right) \equiv \operatorname{Sym}\left(\aleph_{\beta}\right)$;

б) $M_{\alpha} \equiv_{2} M_{\beta}$.

В заключение раздела приведем ряд известных результатов о теориях симметрических полугрупп и групп в иных элементарных и неэлементарных языках.

Теорема 3.2.2 (В.В. Маевский [58]). Границы разрешимости любой бесконечной симметрической полугруппь и класса всех бесконечных симметрических полугрупп в иерархии $S A$ равнь $\{\forall \exists, \exists \forall, \forall \neg \vee, \exists \neg \wedge\}$.

ТЕОРема 3.2.3 (В.В. Маевский [57]). Для любой бесконечной симметрической группьи и для класса всех бесконечных симметрических групп теории $\forall \neg \vee, \exists \neg \wedge$ являются критическими в иерархии $S A$, а теории $\forall \exists \forall$ и $\exists \forall \exists$ являются неразрешимьмми.

Теорема 3.2.4 (Ю. В. Нагребецкая [63]). Для любой бесконечной симметрической группь и класса всех бесконечных симметрических групп $\exists \forall \wedge \vee$-теория разрешима.

Отметим также доказанную в работе [72] элиминируемость кванторов $Q_{0}$ ("существует бесконечно много") и $Q_{1}$ ("существует несчетно много") в элементарном языке на классе симметрических групп.

\section{§4. Производные структуры булевых алгебр}

4.1. Группы автоморфизмов булевых алгебр. Хорошо известна тесная связь теории булевых алгебр с теорией множеств. Тем самьм, не вызывает удивления, что, в отличие от приведенных ранее результатов об элементарных теориях производных структур для групп, колец, графов и т. д., доказательство ряда подобных результатов для булевых алгебр использует такие известные теоретико-множественные предположения, как $V=L, \diamond_{\aleph_{1}}$ и прочие. Исследование груп автоморфизмов булевых алгебр представляет традиционньй интерес. Среди результатов по элементарным теориям групп автоморфизмов булевых алгебр прежде всего укажем на своего рода теорему Левенгейма-Сколема.

Теорема 4.1.1 ( $\diamond_{\aleph_{1}}$ ) (М. Рубин, С. Шелах [93]). Для любой бесконечной булевой алгебрь $\mathfrak{B}$ существует булева алгебра $\mathfrak{B}_{1}$ такая, что $\mid$ Aut $\mathfrak{B}_{1}|\leqslant| \mathfrak{B}_{1} \mid=\aleph_{1} u$ Aut $\mathfrak{B}_{1} \equiv$ Aut $\mathfrak{B}$.

На самом деле в этой теореме доказано даже большее: элементарно эквивалентны соответствуюшие двухосновные алгебраические системы, состоящие из булевой алгебры и группы ее автоморфизмов с операцией действия автоморфизма на булеву алгебру.

Заметим, что этот результат не может быть усилен с заменой кардинала $\aleph_{1}$ на кардинал $\aleph_{0}$. К примеру, в работе М. Рубина [92] построены элементарные формулы $\varphi_{1}$ и $\varphi_{2}$ групповой сигнатуры такие, что:

1) для любой атомной булевой алгебры $\mathfrak{B}$ условия “Aut $\mathfrak{B} \models \varphi_{1}$ " и " $\mathfrak{B}$ - счетна" эквивалентны;

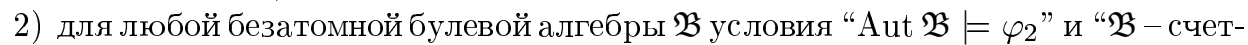
на" эквивалентны. 
Таким образом, Aut-число Левенгейма для многообразия булевых алгебр равно $\aleph_{1}$ (в предположении $\diamond_{\aleph_{1}}$ ).

Этот результат естественным образом инициирует интерес к вопросам определимости счетных булевых алгебр элементарными свойствами их групп автоморфизмов. При решении последнего, столь же естественным образом, возникает ряд ограничений. К примеру, так как для безатомной булевой алгебры $\mathfrak{B}$ и булевой алгебры $\mathfrak{B}_{1}$, получаемой добавлением к $\mathfrak{B}$ одного атома, имеет место изоморфизм Aut $\mathfrak{B} \cong$ Aut $\mathfrak{B}_{1}$, то в вопросе определимости счетной булевой алгебры элементарньми свойствами ее групшы автоморфизмов возникает естественное ограничение $|\operatorname{At}(\mathfrak{B})| \neq 1$. Здесь $\operatorname{At}(\mathfrak{B})$ - совокупность атомов булевой алгебры $\mathfrak{B}$.

Пусть $\mathfrak{C}$ - класс счетных булевых алгебр $\mathfrak{B}$ таких, что $|\operatorname{At}(\mathfrak{B})| \neq 1$. Тогда имеет место следующее утверждение.

Теорема 4.1.2 (М. Рубин [92]). Для любых счетных булевьх алгебр $\mathfrak{B}_{1}$ и $\mathfrak{B}_{2}$ из класса $\mathfrak{C}$ элементарная эквивалентность Aut $\mathfrak{B}_{1} \equiv$ Aut $\mathfrak{B}_{2}$ влечет элементарную әквивалентность $\mathfrak{B}_{1} \equiv \mathfrak{B}_{2}$.

Более того, для любой полной элементарной теории $T$ булевых алгебр существует элементарная формула $\varphi_{T}$ групповой сигнатуры такая, что для любой счетной булевой алгебры $\mathfrak{B}$ из класса $\mathfrak{C}$ условия $\mathfrak{B} \models T$ и Aut $\mathfrak{B} \models \varphi_{T}$ эквивалентны. Иначе говоря, это утверждение имеет место для любого типа элементарной теории $T$, отличного от $\langle 1,1,1\rangle$, и любой счетной булевой алгебры $\mathfrak{B}$.

Как и ранее, через $\equiv_{2}$ обозначим отношение эквивалентности алгебраических систем (совпадение их теорий) в полной логике второго порядка - в логике, допускающей навешивание кванторов $\forall$ и $\exists$ по произвольным предикатам на основном множестве системы.

Теорема 4.1.3 (М. Рубин [92]). Для любьх счетных булевьх алгебр $\mathfrak{B}_{1}$ и $\mathfrak{B}_{2}$, обладающих максимальными атомными әлементами и таких, что $\left|\operatorname{At}\left(\mathfrak{B}_{i}\right)\right| \neq 1$, условия Aut $\mathfrak{B}_{1} \equiv$ Aut $\mathfrak{B}_{2}$ и $\mathfrak{B}_{1} \equiv_{2} \mathfrak{B}_{2}$ равносильньл.

Заметим, что, тем самым, в частности, для любых подобных булевых алгебр $\mathfrak{B}_{1}$ и $\mathfrak{B}_{2}$ имеют место импликации:

$$
\begin{aligned}
& \text { Aut } \mathfrak{B}_{1} \equiv \text { Aut } \mathfrak{B}_{2} \Rightarrow \operatorname{Sub} \mathfrak{B}_{1} \equiv \operatorname{Sub} \mathfrak{B}_{2}, \\
& \text { Aut } \mathfrak{B}_{1} \equiv \text { Aut } \mathfrak{B}_{2} \Rightarrow \operatorname{Con} \mathfrak{B}_{1} \equiv \operatorname{Con} \mathfrak{B}_{2}, \\
& \text { Aut } \mathfrak{B}_{1} \equiv \text { Aut } \mathfrak{B}_{2} \Rightarrow \operatorname{End} \mathfrak{B}_{1} \equiv \text { End } \mathfrak{B}_{2} .
\end{aligned}
$$

В силу известного результата В. Марека [54], [55]: в предположении $V=L$ для любых счетных алгебраических систем $\mathfrak{A}_{1}$ и $\mathfrak{A}_{2}$ конечной сигнатуры из отношения $\mathfrak{A}_{1} \equiv{ }_{2} \mathfrak{A}_{2}$ следует изоморфизм систем $\mathfrak{A}_{1}$ и $\mathfrak{A}_{2}$, из теоремы 4.1.3 вытекает следуюшее утверждение.

Теорема 4.1.4 ( $V=L)$ (М. Рубин [92]). Для любой счетной булевой алгебры $\mathfrak{B}_{1}$, обладающей максимальным атомным әлементом и такой, что $\left|\operatorname{At}\left(\mathfrak{B}_{1}\right)\right| \neq 1$, и любой счетной булевой алгебрь $\mathfrak{B}_{2}$ такой, что $\left|\operatorname{At}\left(\mathfrak{B}_{2}\right)\right| \neq 1$, әлементарная әквивалентность Aut $\mathfrak{B}_{1} \equiv$ Aut $\mathfrak{B}_{2}$ влечет изоморфизм алгебр $\mathfrak{B}_{1}$ u $\mathfrak{B}_{2}$

Без ограничения на принадлежность булевых алгебр классу $\mathfrak{C}$ имеет место следующее утверждение. 
Tеорема $4.1 .5(V=L)$ (М. Рубин [92]). Для любых счетных булевьх алгебр $\mathfrak{B}_{1}$ и $\mathfrak{B}_{2}$ әлементарная әквивалентность Aut $\mathfrak{B}_{1} \equiv$ Aut $\mathfrak{B}_{2}$ влечет изоморфизм Aut $\mathfrak{B}_{1} \cong$ Aut $\mathfrak{B}_{2}$.

Большая часть этих результатов доказьвается путем элементарной интерпреташии в группе Aut $\mathfrak{B}$ двухосновной алгебраической системы $\langle\mathfrak{B}$, Aut $\mathfrak{B} ;$ о, . . $\rangle$, состоящей из булевой алгебры $\mathfrak{B}$ и ее группы автоморфизмов Aut $\mathfrak{B}$ с операцией о действия автоморфизмов на булеву алгебру $(\circ(g, a)=b \Leftrightarrow g \in$ Aut $\mathfrak{B}, a, b \in \mathfrak{B}$ и $g(a)=b)$ и с некоторыми дополнительными предикатами.

Пусть $\mathfrak{C}_{2}-$ класс всех насьшенных булевых алгебр, $\mathfrak{C}_{3}-$ класс 1-гомеогенных, $\omega$-насьшенных булевых алгебр $\mathfrak{B}$ таких, что $\left|A t\left(\mathfrak{B}^{(n)}\right)\right| \neq 1$. Напомним, что булева алгебра $\mathfrak{B}$ 1-гомогенна тогда и только тогда, когда ее элементы, имеюшие один и тот же элементарньй тип, переводятся друг в друга каким-либо автоморфизмом алгебры $\mathfrak{B}$, а $\mathfrak{B}^{(n)}$ - это $n$-я производная Ершова-Тарского булевой алгебры $\mathfrak{B}$. Пусть $\mathfrak{C}_{1}=\mathfrak{C}_{2} \cup \mathfrak{C}_{3}$.

Среди других результатов об элементарных теориях групп автоморфизмов булевых алгебр отметим следуюший.

Теорема 4.1.6 (М. Рубин [91]). Для любых булевых алгебр $\mathfrak{B}_{1}$ и $\mathfrak{B}_{2}$ из класса $\mathfrak{C}_{1}$ элементарная эквивалентность Aut $\mathfrak{B}_{1} \equiv$ Aut $\mathfrak{B}_{2}$ влечет элементарную әквивалентность двухосновных систем $\left\langle\mathfrak{B}_{1}\right.$, Aut $\mathfrak{B}_{1}$, o $\rangle \equiv\left\langle\mathfrak{B}_{2}\right.$, Aut $\mathfrak{B}_{2}$, о $\rangle$.

Ограничения в условиях приведенных выше теорем классами $\mathfrak{C}_{1}$ и счетных булевых алгебр весьма сушественны. Действительно, как хорошо известно [20], в предположении обобщенной континуум-гипотезы существуют эндожесткие (т.е. не имеюшие нетривиальных инъективных эндоморфизмов) $k$-плотные булевы алгебры $\mathfrak{B}$ любой несчетной мощности $k$. Тем самым, для любой булевой алгебры $\mathfrak{A}$ имеет место Aut $\mathfrak{A} \cong$ Aut $\mathfrak{A} \times \mathfrak{B}$. То есть Aut-число Ханфа для многообразия булевых алгебр равно $\aleph_{0}$ (в предположении обобшенной континуум-гипотезы).

Отметим, что фактически не проводилось изучение теорий групп автоморфизмов булевых алгебр в иных, отличных от элементарного $\mathscr{E}$, языках.

Среди открытых вопросов, связанных с элементарными теориями групп автоморфизмов булевых алгебр, укажем на следуюший:

нахождение классификации свободных булевых алгебр на основе элементарной эквивалентности их груп автоморфизмов (см. $\S 6)$.

Из известного результата Ю. Л. Ершова [30] о наследственной неразрешимости элементарной теории класса всех конечных симметрических групп очевидным образом вытекает следуюший критерий разрешимости элементарных теорий групп автоморфизмов булевых алгебр:

для любой булевой алгебры $\mathfrak{B}$ элементарная теория группы Aut $\mathfrak{B}$ разрешима тогда и только тогда, когда $\mathfrak{B} \cong \mathfrak{B}_{1} \times \mathfrak{B}_{2}$, где $\mathfrak{B}_{1}$ - конечная, а $\mathfrak{B}$ - безатомная эндожесткая булева алгебра.

В связи с этим, отметим и результат В. И. Мартьянова [56] о неразрешимости элементарной теории класса всех булевых алгебр в сигнатуре $\sigma=\langle\wedge, \vee, \neg, 0,1, f\rangle$, где одноместная функция $f$ интепретируется произвольным автоморфизмом булевой алгебры. 
4.2. Решетки конгруэнций и подалгебр булевых алгебр. Как хорошо известно, решетки конгруэнций булевых алгебр изоморфны решеткам их идеалов (фильтров). Главные идеалы выделяются в решетке идеалов булевых алгебр свойством “иметь дополнение". Тем самьм, в решетке Con $\mathfrak{B}$ любой булевой алгебры $\mathfrak{B}$ диофантовыми формулами интепретируется двухосновная алгебраическая система $\langle\mathfrak{B}, \operatorname{Con} \mathfrak{B}, \in\rangle$, состоящая из булевой алгебры $\mathfrak{B}$, решетки Con $\mathfrak{B}$ и отношения $\in$ такого, что $\in(a, b, c)$ имеет место тогда и только тогда, когда $a, b \in \mathfrak{B}, c \in \operatorname{Con} \mathfrak{B}$ и $\langle a, b\rangle \in c$. Таким образом, в частности, для любых булевых алгебр имеет место импликация Con $\mathfrak{B}_{1} \equiv \operatorname{Con} \mathfrak{B}_{2} \Rightarrow \mathfrak{B}_{1} \equiv \mathfrak{B}_{2}$.

Хорошо известны следуюшие результаты М. Рабина [87] и Л. Хайндорфа [42] по вопросу разрешимости теорий второго порядка булевых алгебр с кванторами по идеалам. Мы сформулируем здесь эти результаты как результаты об элементарных теориях решеток конгруэнщий булевых алгебр (что в силу замеченного вьше равносиль$\mathrm{HO})$.

Теорема 4.2 .1 (М. Рабин [87]). Элементарная теория класса решеток $\{$ Con $\mathfrak{B} \mid \mathfrak{B}$ - счетная либо конечная булева алгебра $\}$ разрешима.

Теорема 4.2.2 (Л. Хайндорф [42]). Элементарная теория каждого из следующих классов решеток неразрешима:

$\{$ Con $\mathfrak{B} \mid \mathfrak{B}$ - несчетная булева алгебра $\}$,

$\{$ Con $\mathfrak{B} \mid \mathfrak{B}$ - произвольная булева алгебра $\}$,

$\{$ Con $\mathfrak{B} \mid \mathfrak{B}$ - суператомная булева алгебра $\}$.

В работе [85] построена элементарная интепретация двухосновной алгебраической системы $\langle\mathfrak{B}, \operatorname{Sub} \mathfrak{B}, \in\rangle$ в решетке Sub $\mathfrak{B}$ для любой атомной булевой алгебры $\mathfrak{B}$. Здесь система $\langle\mathfrak{B}, \operatorname{Sub} \mathfrak{B}, \in\rangle$ состоит из булевой алгебры $\mathfrak{B}$, решетки Sub $\mathfrak{B}$ ее подалгебр, а отношение $\in(a, b)$ имеет место тогда и только тогда, когда $a \in \mathfrak{B}, b \in \operatorname{Sub} \mathfrak{B}$ и $a \in b$. В силу этого и известного результата М. Рубина [90] о неразрешимости элементарной теории класса булевых пар $\left\langle\mathfrak{B}_{1}, \mathfrak{B}_{2}\right\rangle$ (булева алгебра $\mathfrak{B}_{1}$ с выделенной подалгеброй $\mathfrak{B}_{2}$ ) для атомных булевых алгебр $\mathfrak{B}_{1}$ имеет место следующее утверждение.

Теорема 4.2.3 (С. Баррис, Г. П. Санкапанавар [26]). Элементарные теории следующих классов решеток неразрешимы:

$\{\operatorname{Sub} \mathfrak{B} \mid \mathfrak{B}-$ произвольная булева алгебра $\}$,

$\{\mathrm{Sub} \mathfrak{B} \mid \mathfrak{B}$ - атомная булева алгебра $\}$,

$\{\operatorname{Sub} \mathfrak{B} \mid \mathfrak{B}-$ безатомная булева алгебра $\}$.

На самом деле, имеет место более сильньй результат.

Tеорема 4.2.4 (А. Г. Пинус, З.А. Дулатова [82]). Для класса решеток $\{\mathrm{Sub} \mathfrak{B}$ | $\mathfrak{B}$ - произвольная булева алгебра $\forall \forall \forall \exists \forall \exists \forall \theta$-теория неразрешима, $a \forall \theta$-теория разрешима. Для класса решеток конечных подалгебр произвольных булевьх алгебр $\forall \exists \forall \exists \forall \theta$-теория неразрешима.

Среди открытых вопросов, связанных с элементарными теориями решеток конгруэнций и подалгебр булевых алгебр, остаются не разработанными вопросы нахождения гранищ разрешимости классов решеток $\{$ Con $\mathfrak{B} \mid \mathfrak{B}$ - произвольная булева алгебра $\}$, $\{\operatorname{Sub} \mathfrak{B} \mid \mathfrak{B}-$ произвольная булева алгебра $\}$ в различных иерархиях языков логики первого порядка. 
Отметим также вопрос описания пар булевых алгебр $\mathfrak{B}_{1}, \mathfrak{B}_{2}$, удовлетворяющих импликации $\operatorname{Sub} \mathfrak{B}_{1} \equiv \operatorname{Sub} \mathfrak{B}_{2} \Rightarrow \mathfrak{B}_{1} \equiv \mathfrak{B}_{2}$; выше было отмечено, что импликация имеет место для любых атомных булевых алгебр.

В заключение заметим, что с помощью булевых конструкций (булевых степеней и булевых произведений) целый ряд перечисленных вьше результатов, по вопросам разрешимости теорий, может быть перенесен со случая булевых алгебр на случай дискриминаторных или даже произвольных конгруэнщ-дистрибутивных многообразий.

Теорема 4.2 .5 (А.Г. Пинус [76]). 1) Для любого нетривиального дискриминаторного многообразия $V \quad \forall \exists \forall \exists \forall \exists \forall \theta$-теория класса решеток $\{\operatorname{Sub} \mathfrak{A} \mid \mathfrak{A} \in V\}$ неразрешима.

2) Для любого дискриминаторного многообразия $V \quad \forall \theta$-теория класса решеток $\{\operatorname{Sub} \mathfrak{A} \mid \mathfrak{A} \in V\}$ разрешима.

3) Для любого нетривиального конгруэни-дистрибутивного многообразия $V$ элементарная теория класса решеток $\{$ Con $\mathfrak{A} \mid \mathfrak{A} \in V\}$ неразрешима.

4) Для любого конечно порохсденного дискриминаторного многообразия $V$ әлементарная теория класса решеток $\{\operatorname{Con} \mathfrak{A} \mid \mathfrak{A}$ - счетная $V$-алгебра $\}$ разрешима.

Близкие вопросы рассматриваются в работе Л. Хайндорфа [41].

\section{§. Производные структуры свободных алгебр}

Единственным инвариантом бесконечно порожденных свободных алгебр $\mathscr{F} V(k)$ данного многообразия $V$ является мошность $k$ свободных порождающих. При этом все подобные алгебры $\mathscr{F} V(k)$, независимо от кардинала $k$, имеют одинаковое локальное строение, в силу чего все они элементарно эквивалентны между собой (неразличимы на языке логики первого порядка). Совершенно иная ситуащия складывается при изучении производных структур свободных алгебр $\mathscr{F}_{V}(k)$, в частности, полугруш их эндоморфизмов End $\mathscr{F}_{V}(k)$, решеток их конгруэнщий $\operatorname{Con} \mathscr{F}_{V}(k)$, решеток их подалгебр $\operatorname{Sub} \mathscr{F}_{V}(k)$, групп их автоморфизмов Aut $\mathscr{F}_{V}(k)$. Отношение элементарной эквивалентности для каждой пары этих производных структур свободных алгебр индуцирует некоторое отношение эквивалентности на классе всех бесконечных кардиналов:

$$
\begin{aligned}
k & \equiv{ }_{\text {End }}^{V} \lambda \Leftrightarrow \operatorname{End} \mathscr{F}_{V}(k) \equiv \operatorname{End} \mathscr{F}_{V}(\lambda), \\
k & \equiv_{\text {Con }}^{V} \lambda \Leftrightarrow \operatorname{Con} \mathscr{F}_{V}(k) \equiv \operatorname{Con} \mathscr{F}_{V}(\lambda), \\
k & \equiv_{\text {Sub }}^{V} \lambda \Leftrightarrow \operatorname{Sub} \mathscr{F}_{V}(k) \equiv \operatorname{Sub} \mathscr{F}_{V}(\lambda), \\
k & \equiv_{\text {Aut }}^{V} \lambda \Leftrightarrow \operatorname{Aut} \mathscr{F}_{V}(k) \equiv \text { Aut } \mathscr{F}_{V}(\lambda) .
\end{aligned}
$$

Мы ограничимся здесь рассмотрением лишш конечно базируемых многообразий $V$ конечной сигнатуры, с тем чтобы для любого бесконечного кардинала $k V$-свободная $k$-порожденная алгебра $\mathscr{F}_{V}(k)$ могла быть определена на множестве $k$ формулами логики второго порядка. Тем самьм, при рассмотрении лишш конечно базируемых многообразий конечной сигнатуры данные отношения эквивалентности на классе бесконечных кардиналов сводятся к совпадению совокупностей истинных на кардиналах $k$ и $\lambda$ формул логики второго порядка некоторого фиксированного вида.

Классификация некоторых фрагментов логики второго порядка получена в работе С. Шелаха [95] (см. также [2]) при изучении расширений логики первого порядка, 
допускаюших навешивание кванторов по предикатам $P$, удовлетворяюшим той или иной фиксированной формуле $\phi$ логики первого порядка сигнатуры $\langle P\rangle$. При этом в работе [95] доказано, что выразительные возможности любой подобной логики (зависяшей от выбора формулы $\phi$ ) совпадают с выразительными возможностями одной из следуюших четырех логик данного типа:

1) логики первого порядка - УИП;

2) монадической логики - логики второго порядка с кванторами по произвольньгм одноместньм предикатам - Mon;

3) симметрической логики - логики второго порядка с кванторами по двухместньм предикатам, соответствующим произвольньм перестановкам на основном множестве, - Sym;

4) полной логики второго порядка - логике второго порядка с кванторами по произвольным предикатам на основном множестве - 2 ИП.

Заметим, что совпадение теорий бесконечных кардиналов в первых двух из перечисленных логик индуцирует на совокупности всех бесконечных кардиналов одно и то же отношение $\nabla$ (истинное на любой паре бесконечных кардиналов). Совпадение теорий бесконечных кардиналов в логике Sym индуцирует на классе всех бесконечных кардиналов отношение эквивалентности $\equiv_{p}$, совпадающее с отношением элементарной эквивалентности соответствуюших симметрических груп и описанное в теореме 3.2.1. Наконец, как и ранее, через $\equiv_{2}$ будем обозначать отношение эквивалентности на классе всех бесконечных кардиналов, индуцированное совпадением теорий этих кардиналов в логике 2 ИП.

В силу замеченного вьше ограничение рассмотрением лишш конечно базируемых многообразий $V$ конечной сигнатуры влечет справедливость следующей импликации:

$$
k \equiv_{2} \lambda \Rightarrow k \equiv{ }_{\text {End }}^{V} \lambda \& k \equiv_{\text {Sub }}^{V} \lambda \& k \equiv{ }_{\text {Aut }}^{V} \lambda \& k \equiv{ }_{\text {Con }}^{V} \lambda .
$$

Во всех известных к настоящему времени случаях для любого конечно базируемого многообразия $V$ конечной сигнатуры отношения $\equiv_{\text {End }}^{V}, \equiv_{\text {Con }}^{V}, \equiv_{\text {Aut }}^{V}, \equiv_{\text {Sub }}^{V}$ совпадают с одним из отношений $\nabla, \equiv_{p}$ либо $\equiv_{2}$.

Вопрос об элементарной эквивалентности полугруп эндоморфизмов свободных алгебр решен в работе С. Шелаха (для рассматриваемых многообразий - конечно базируемых, конечной сигнатуры и кардиналов - бесконечных).

Теорема 5.1 (С. Шелах [99]). Отношение $\equiv_{\text {End }}^{V}$ совпадает с отношением $\equiv_{2}$.

В качестве примера ситуашии с отношениями $\equiv_{\mathrm{Sub}}^{V}, \equiv_{\mathrm{Aut}}^{V}, \equiv_{\mathrm{Con}}^{V}$ рассмотрим случай многообразий множеств (алгебр пустой сигнатуры). В этом случае:

Sub $\mathscr{F}_{V}(k)=P(k)$ - решетка подмножеств кардинала $k$;

Aut $\mathscr{F}_{V}(k)=\operatorname{Sym}(k)$ - симметрическая группа на кардинале $k$;

Con $\mathscr{F}_{V}(k)=\operatorname{Part}(k)$ - решетка разбиений кардинала $k$.

А. Тарским [108] была доказана элементарная эквивалентность любых бесконечных атомных булевых алгебр. По поводу элементарной эквивалентности симметрических групш см. теорему 3.2.1.

А. Г. Пинусом [75] доказана элементарная интерпретируемость полной логики второго порядка 2 ИП на кардинале $k$ в решетке $\operatorname{Part}(k)$. В силу этих результатов имеет место следуюшая теорема. 
Теорема 5.2 (А. Тарский [108], Ю. Л. Ершов [30], С. Шелах [96], А. Г. Пинус [75]). Для многообразия $V$ множеств отношение $\equiv_{\mathrm{Sub}}^{V}$ совпадает с отношением $\nabla$, отношение $\equiv_{\mathrm{Aut}}^{V}-c$ отношением $\equiv_{p}$, отношение $\equiv_{\mathrm{Con}}^{V}-c$ отношением $\equiv_{2}$.

Таким образом, многообразие множеств в качестве тройки отношений $〈 \equiv{ }_{\mathrm{Sub}}^{V}$, $\left.\equiv_{\text {Aut }}^{V}, \equiv_{\text {Con }}^{V}\right\rangle$ на классе всех бесконечных кардиналов реализует тройку отношений $\left\langle\nabla, \equiv_{p}, \equiv_{2}\right\rangle$. (О близких результатах см. также работу [68].)

Столь же досконально изучена ситуация с отношениями $\equiv_{\mathrm{Sub}}^{V}, \equiv_{\mathrm{Aut}}^{V}$ и $\equiv_{\mathrm{Con}}^{V}$ для многообразий векторных пространств, см. теоремы раздела 1.2:

Теорема 5.3 (О. В. Белеградек, В. Толстых [19]). Для многообразия $V$ векторных пространств над любым фиксированным конечным полем (над полями $\mathbb{Q}$, $\mathbb{R}, \mathbb{C}$ и др.) имеет место равенство

$$
\left\langle\equiv_{\text {Sub }}^{V}, \equiv_{\text {Aut }}^{V}, \equiv_{\text {Con }}^{V}\right\rangle=\left\langle\equiv_{2}, \equiv_{2}, \equiv_{2}\right\rangle .
$$

Этот результат позволяет получить частичную информацию об отношениях $\equiv_{\mathrm{Con}}^{V}$, $\equiv V_{\text {Sub }}$ для многообразий групш.

Для любого многообразия $V$ через $F V_{\text {Sub }}\left(F V_{\text {Con }}\right)$ обозначим класс решеток вида $\operatorname{Sub} \mathscr{F}_{V}(k)\left(\operatorname{Con} \mathscr{F}_{V}(k)\right)$ для любого бесконечного кардинала $k$.

Алгебру $\mathfrak{A}$ из класса $\mathfrak{K}$ назовем конечно элементарно аксиоматизируемой в классе $\mathfrak{K}$, если существует формула $\varphi$ логики первого порядка такая, что для любой алгебры $\mathfrak{B}$ из $\mathfrak{K}$ условия $\mathfrak{B} \models \varphi$ и $\mathfrak{B} \cong \mathfrak{A}$ равносильны. Кардинал $k$ назовем конечно аксиоматизируемым в логике второго порядка, если существует формула $\varphi$ полной логики второго порядка такая, что $\{k\}=\{\lambda$-кардинал $\mid \lambda \models \varphi\}$. Очевидным образом, вопрос об элементарной определимости решетки Sub $\mathscr{F}_{V}(k)\left(\operatorname{Con} \mathscr{F}_{V}(k)\right)$ в классе $F V_{\text {Sub }}\left(F V_{\text {Con }}\right)$ связан с вопросами описания отношения $\equiv_{\text {Sub }}^{V}\left(\equiv_{\text {Con }}^{V}\right)$ : подобная определимость решетки $\operatorname{Sub} \mathscr{F}_{V}(k)\left(\operatorname{Con} \mathscr{F}_{V}(k)\right)$ в класcе $F V_{\text {Sub }}\left(F V_{\text {Con }}\right)$ влечет одноэлементность класса $\equiv \mathrm{Sub}^{V}\left(\equiv_{\mathrm{Con}^{-}}^{V}\right)$ эквивалентности для кардинала $k$.

Теорема 5.4 (А.Г. Пинус [81]). а) Для любого нетривиального многообразия $V$ групп решетка Con $\mathscr{F}_{V}(k)$ конечно әлементарно аксиоматизируема в классе ре-

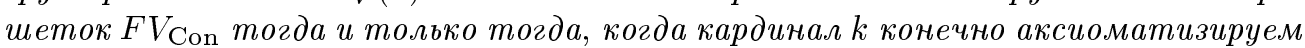
в полной логике второго порядка.

б) Для любого нетривиального многообразия $V$ абелевых групп решетка Sub $\mathscr{F}_{V}(k)$ конечно әлементарно аксиоматизируема в классе решеток $F V_{\mathrm{Sub}}$ тогда и только тогда, когда кардинал $k$ конечно аксиоматизируем в полной логике второго порядка.

Отметим еще известные результаты относительно рассматриваемых эквивалентностей на классе бесконечных кардиналов, связанные с группами. (См. также работу [112].)

Теорема 5.5. а) (В. Толстых [110].) Для многообразия $V$ всех групп отношение ${ }_{\text {Aut }}^{V}$ совпадает с отношением $\equiv_{2}$.

б) (В. Толстых [113].) Для любого многообразия нильпотентных групп $\mathfrak{N}_{c}$ ступени $c \geqslant 2$ отношение $\equiv_{\mathrm{Aut}}^{\mathfrak{N}_{c}}$ совпадает с отношением $\equiv_{2}$.

в) (В. Толстых [114].) Для многообразия $V$ всех абелевых групп отношения $\equiv_{\text {Aut }}^{V} u \equiv_{2}$ совпадают.

Частично прояснена информация об эквивалентностях $\equiv_{\mathrm{Sub}}^{V}, \equiv_{\mathrm{Con}}^{V}$ для нетривиальных многообразий $V$ унаров. 
Теорема 5.6 (А.Г. Пинус [81]). Для любого нетривиального многообразия $V$ унаров:

а) отношение $\equiv_{\mathrm{Sub}}^{V}$ совпадает с отношением $\nabla$;

б) для любого бесконечного кардинала $k$ решетка $\operatorname{Con} \mathscr{F}_{V}(k)$ конечно әлементарно аксиоматизируема в классе решеток $F V_{\text {Соn }}$ тогда и только тогда, когда кардинал $k$ конечно аксиоматизируем в полной логике второго порядка.

Вопрос об отношении $\equiv{ }_{\text {Aut }}^{V}$ для многообразий $V$ унаров (совпадение этого отношения с отношением $\equiv_{p}$ ) упирается в следуюший открытьй теоретико-групповой вопрос: верно ли, что для любой счетной групы $G$ и любых бесконечных кардиналов $k$ и $\lambda$ элементарная эквивалентность груп $\operatorname{Sym}(k)$ и $\operatorname{Sym}(\lambda)$ влечет элементарную эквивалентность групп $G S \operatorname{Sym}(k)$ и $G S \operatorname{Sym}(\lambda)$ ? Здесь $G_{1} S G_{2}$ - полное сплетение групп $G_{1}$ и $G_{2}$.

Напомним, что многообразие $V$ назьвается нормальным, если для любого нетривиального терма $t(\bar{x})$ сигнатуры этого многообразия и любой переменной $y$ тождество $t(\bar{x})=y$ не является истинным на $V$.

Многообразие $V$ обладает свойством исключения, если для любого кардинала $k$ и любого $\alpha \in k$ подмножество $\mathscr{F}_{V}(k) \backslash\{\alpha\}$ является подалгеброй алгебры $\mathscr{F}_{V}(k)$.

Многообразие $V$ обладает существенно двухместной функиией, если существует сигнатурная функция $f(x, y)$ такая, что тождества $f(x, y)=f(x, z)$ и $f(y, x)=$ $f(z, x)$ не является истинными на $V$.

Многообразие $V$ идемпотентно, если для любой сигнатурной функции $g\left(x_{1}\right.$, $\left.\ldots, x_{n}\right)$ на $V$ истинны тождества $g(x, \ldots, x)=x$.

Многообразие $V$ называется 2 -конечныл , если конечна алгебра $\mathscr{F} V(2)$.

Заметим, что, к примеру, свойством исключения обладает любое многообразие решеток, примерами многообразий со свойством исключения являются также любое многообразие директоидов, многообразие полурешеток, любое нормальное многообразие.

Теорема 5.7 (А.Г. Пинус, Г. Роуз [84]). Для любого идемпотентного 2-конечного многообразия $V$ со свойством исключения, обладающего существенно двухместной функчией, отношение $\equiv_{\mathrm{Sub}}^{V}$ совпадает с отношением $\equiv_{2}$.

Условия этой теоремы выполнены для многообразия полурешеток, любого многообразия решеток, коммутативных директоидов.

Теорема 5.8 (А.Г. Пинус, Г. Роуз [85]). Для любого нормального многообразия $V$ решетка Con $\mathscr{F}_{V}(k)$ конечно әлементарно аксиоматизируема в классе

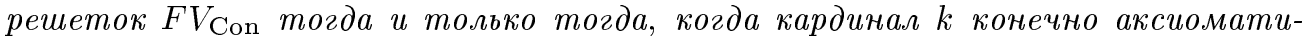
зируем в полной логике второго порядка.

Для ряда нормальных многообразий утверждение теоремы 5.8 может быть усилеHO.

Через (*) обозначим следующее свойство многообразия $V$ : понятие $\vee$-неразложимой конгруэнщии и понятие главной конгруэнции на $V$-свободных алгебрах $\mathscr{F} V(k)$ совпадают. В частности, свойством (*) обладает многообразие всех полугрупп и многообразие всех коммутативных полугрупп. 
Теорема 5.9 (А.Г. Пинус [81]). Для любого нетривиального нормального многообразия $V$ со свойством $(*)$ отношения $\equiv_{\text {Con }}^{V} u \equiv_{2}$ совпадают.

Тем самым, совпадают отношения $\equiv_{\text {Con }}^{V}$ и $\equiv_{2}$, в частности, для многообразий всех полугрупп, всех коммутативных полугрупп.

Аналогичная ситуация имеет место для многообразия $V$ (всех коммутативных) полугруп и отношения $\equiv \stackrel{\text { Sub}}{V}$.

Теорема 5.10 (А.Г. Пинус [81]). Для многообразия $V$ всех (всех коммутативных ) полугрупп отношение $\equiv_{\mathrm{Sub}}^{V}$ совпадает с отношением $\equiv_{2}$.

Заметим также, что, очевидным образом, отношения $\equiv_{\mathrm{Aut}}^{V}$ и $\equiv_{p}$ совпадают для любого нормального многообразия $V$. Тем самьм, полностью известна тройка отношений $\left\langle\equiv_{\text {Sub }}^{V}, \equiv_{\text {Aut }}^{V}, \equiv_{\text {Con }}^{V}\right\rangle=\left\langle\equiv_{2}, \equiv_{p}, \equiv_{2}\right\rangle$ для многообразия $V$ всех полугрупп (всех коммутативных полугрупп).

ТеОрема 5.11 (А.Г. Пинус[81]). Для многообразия $V$ полурешеток отношения $\equiv_{\text {Con }}^{V} u \equiv_{2}$ совпадают.

Таким образом, для многообразия $V$ полурешеток тройка отношений $\left\langle\equiv{ }_{\mathrm{Sub}}^{V}, \equiv_{\mathrm{Aut}}^{V}\right.$, $\left.\equiv_{\text {Соn }}^{V}\right\rangle$ совпадает с тройкой $\left\langle\equiv_{2}, \equiv_{p}, \equiv_{2}\right\rangle$.

Здесь равенство отношений $\equiv_{\text {Aut }}^{V}$ и ${ }_{p}$ следует из того, что единственными $\wedge$-неразложимьми элементами полурешетки $\mathscr{F}_{V}(k)$ являются ее свободные порождаюшие.

Напомним, что тождество $t_{1}=t_{2}$ назьвается регулярным, если термы $t_{1}$ и $t_{2}$ зависят от одних и тех же переменных. Многообразие, аксиоматизируемое некоторой совокупностью регулярных тождеств, назьвается регулярны.м.

Из теоремы 5.11 вытекает следуюшее утверждение.

Теорема 5.12 (А.Г. Пинус [81]). Для любого регулярного многообразия $V$ решетка Con $\mathscr{F}_{V}(k)$ конечно элементарно аксиоматизируема в классе решеток

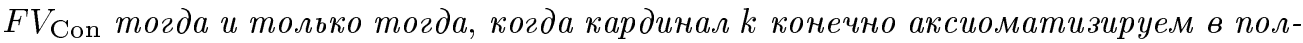
ной логике второго порядка.

Суммируем приведенные выше факты, связанные со строением отношений $\equiv_{\mathrm{Sub}}^{V}$, $\equiv_{\text {Aut }}^{V}, \equiv_{\text {Con }}^{V}$ для различных многообразий $V$ в виде таблицы 1, в которой знак ? означает открытость данного вопроса.

Здесь символ $\equiv_{2}^{*}$, стояший в столбце $\equiv_{\mathrm{Con}}^{V}\left(\equiv_{\mathrm{Sub}}^{V}\right)$ означает, что решетка $\operatorname{Con} \mathscr{F}_{V}(k)$ $\left(\operatorname{Sub} \mathscr{F}_{V}(k)\right)$ конечно элементарно аксиоматизируема в классе $F V_{\mathrm{Con}}\left(F V_{\mathrm{Sub}}\right)$ тогда и только тогда, когда кардинал $k$ конечно аксиоматизируем в полной логике второго порядка.

В связи с приведенными результатами возникает цельй ряд естественных вопросов. Прежде всего: нельзя ли усилить результаты, касающиеся аксиоматизируемости, до совпадения соответствующих отношений эквивалентности?

Совпадают ли отношения $\equiv_{\mathrm{Con}}^{V}$ и $\equiv_{2}\left(\equiv_{\mathrm{Sub}}^{V}\right.$ и $\left.\equiv_{2}\right)$ для любого конечно базируемого многообразия $V$ унаров, груп, всех нормальных многообразий, регулярных многообразий (многообразий групп)?

Как видно из представленной таблицы, все фигурируюшие в качестве отношений $\equiv_{\mathrm{Sub}}^{V}, \equiv_{\mathrm{Aut}}^{V}, \equiv_{\mathrm{Con}}^{V}$ отношения и претендуюшие на эту роль (исходя из элементарно определимых производных структур свободных алгебр) суть отношения одного из видов: $\nabla, \equiv_{p}$ или $\equiv_{2}$. Представляет интерес ответ на вопрос: всегда ли это так? То есть верно ли, что для любого конечного базируемого многообразия $V$ конечной сигнатуры отношения $\equiv_{\mathrm{Sub}}^{V}, \equiv_{\mathrm{Aut}}^{V}$ и $\equiv_{\text {Con }}^{V}$ входят в совокупность $\left\{\nabla, \equiv_{p}, \equiv_{2}\right\}$ ? 
ТАБЛИЦА 1

\begin{tabular}{|c|c|c|c|}
\hline Многообразие $V$ & $\equiv_{\mathrm{Sub}}^{V}$ & $\equiv_{\mathrm{Aut}}^{V}$ & $\equiv{ }_{\text {Con }}^{V}$ \\
\hline 1. Многообразие множеств & $\nabla$ Тарский & $\equiv{ }_{p}$ Ершов & $\equiv_{2}$ Пинус \\
\hline $\begin{array}{l}\text { 2. Многообразие векторных } \\
\text { пространств над конечным полем }\end{array}$ & $\begin{array}{l}\equiv_{2} \text { Белеградек- } \\
\text { Толстьх }\end{array}$ & $\begin{array}{l}\equiv_{2} \text { Белеградек- } \\
\text { Толстых }\end{array}$ & $\begin{array}{l}\equiv_{2} \text { Белеградек- } \\
\text { Толстых }\end{array}$ \\
\hline 3. Многообразие унаров & $\nabla$ Пинус & $?$ & $\equiv_{2}^{*}$ Пинус \\
\hline $\begin{array}{l}\text { 4. Идемпотентные, симметричные } \\
\text { многообразия со свойством } \\
\text { исключения, 2-конечные и } \\
\text { с существенно двухместной функцией } \\
\text { (многообразия решеток, } \\
\text { коммутативных директоидов, } \\
\text { полурешеток) }\end{array}$ & $\equiv_{2}$ Пинус-Роуз & $?$ & $?$ \\
\hline 5. Нормальные многообразия & $?$ & $\equiv p$ очевидно & $\equiv_{2}^{*}$ Пинус-Роуз \\
\hline $\begin{array}{l}\text { 6. Нормальные многообразия, } \\
\text { для которых } \vee \text {-неразложимше } \\
\text { конгруэнции на свободной алгебре } \\
\text { суть главные } \\
\text { (в частности, многообразия } \\
\text { всех полугруп, } \\
\text { всех коммутативных полугрупп) }\end{array}$ & $?$ & $\equiv p$ очевидно & $\equiv_{2}$ Пинус \\
\hline $\begin{array}{l}\text { 7. Многообразие всех полугрупп } \\
\text { (всех коммутативных полугрупп) }\end{array}$ & $\equiv_{2}$ Пинус & $\equiv p$ очевидно & $\equiv_{2}$ Пинус \\
\hline 8. Многообразие полурешеток & $\equiv_{2}$ Пинус-Роуз & $\equiv p$ очевидно & $\equiv_{2}$ Пинус \\
\hline 9. Регулярные многообразия & $?$ & $?$ & $\equiv_{2}^{*}$ Пинус \\
\hline 10. Многообразие всех групп & $\equiv_{2}^{*}$ Пинус & $\equiv_{2}$ Толстых & $\equiv_{2}^{*}$ Пинус \\
\hline $\begin{array}{l}\text { 11. Любое нетривиальное } \\
\text { многообразие групп }\end{array}$ & $?$ & ? & $\equiv_{2}^{*}$ Пинус \\
\hline $\begin{array}{l}\text { 12. Любое нетривиальное } \\
\text { многообразие абелевых групп }\end{array}$ & $\equiv_{2}^{*}$ Пинус & $?$ & $\equiv_{2}^{*}$ Пинус \\
\hline $\begin{array}{l}\text { 13. Многообразие всех } \\
\text { абелевых групп }\end{array}$ & $\equiv_{2}^{*}$ Пинус & $\equiv_{2}$ Толстых & $\equiv_{2}^{*}$ Пинус \\
\hline $\begin{array}{l}\text { 14. Любое многообразие всех } \\
\text { нильпотентных групп ступени } \geqslant 2\end{array}$ & $?$ & $\equiv_{2}$ Толстых & $\equiv_{2}^{*}$ Пинус \\
\hline
\end{tabular}

При этом важно отметить, что все доказательства известных равенств между отношениями $\equiv_{\mathrm{Sub}}^{V}, \equiv_{\mathrm{Aut}}^{V}, \equiv_{\mathrm{Con}}^{V}$ и отношениями $\equiv_{p}, \equiv_{2}$ проходят путем интерпретации соответствуюших логик второго порядка $\mathrm{Sym}$, либо 2 ИП на кардиналах $k$ в элементарных теориях решеток $\operatorname{Con} \mathscr{F}_{V}(k), \operatorname{Sub} \mathscr{F}_{v}(k)$, либо групп Aut $\mathscr{F}_{V}(k)$. Однако подобные интерпретации не могут иметь места для любых многообразий $V$. Действительно, хорошо известны результаты М. Рабина [87] о разрешимости теории второго порядка счетной свободной булевой алгебры с кванторами по фильтрам и Л. Хайндорфа [42] о неразрешимости подобной теории для несчетных свободных булевых алгебр (см. $\S 4$ ). Тем самьм, для многообразия $B A$ булевых алгебр (так как двуосновная модель, состояшая из булевой алгебры и фильтров, как хорошо известно, элементарно интерпретируется в решетке фильтров соответствуюшей булевой алгебры) отношение $\equiv \underset{\text { Con }}{B A}$ не совпадает с отношением $\nabla$. Но в то же время в силу результата М. Рабина 
логики $\mathrm{Sym}$ и 2 ИП на кардинале $\aleph_{0}$ не могут быть проинтерпретированы (в силу их неразрешимости) в элементарной теории решетки Con $\mathscr{F}_{B A}\left(\aleph_{0}\right)$.

Приведенная выше таблица индуцирует также следуюший вопрос. Пусть $\left\langle\equiv^{1}, \equiv^{2}\right.$, $\left.\equiv^{3}\right\rangle$ - произвольный набор из трех эквивалентностей на классе всех бесконечных кардиналов такой, что $\equiv^{i} \in\left\{\nabla, \equiv_{p}, \equiv_{2}\right\}$. Сушествует ли конечно базируемое многообразие $V$ конечной сигнатуры такое, что $\left\langle\equiv_{\mathrm{Sub}}^{V}, \equiv_{\mathrm{Aut}}^{V}, \equiv_{\mathrm{Con}}^{V}\right\rangle=\left\langle\equiv^{1}, \equiv^{2}, \equiv^{3}\right\rangle$ ?

При отрицательном ответе на эту проблему возникает вопрос описания троек эквивалентностей $\left\langle\equiv^{1}, \equiv^{2}, \equiv^{3}\right\rangle$, где $\equiv^{i} \in\left\{\nabla, \equiv_{p}, \equiv_{2}\right\}$, реализуемых в качестве тройки $\left\langle\equiv_{\mathrm{Sub}}^{V}, \equiv_{\mathrm{Aut}}^{V}, \equiv_{\mathrm{Con}}^{V}\right\rangle$ для конечно базируемых многообразий $V$ конечной сигнатуры.

Сушествует ли конечно базируемое многообразие $V$ конечной сигнатуры, для которого вьполнено одно из следуюших условий:

a) отношение $\equiv_{\mathrm{Sub}}^{V}$ совпадает с отношением $\equiv_{p}$;

б) отношение $\equiv{ }_{\text {Aut }}^{V}$ совпадает с отношением $\nabla$;

в) отношение $\equiv_{\text {Con }}^{V}$ совпадает с отношением $\equiv_{p}(\nabla)$ ?

Наконец, естественен интерес к описанию отношений $\equiv_{\mathrm{Sub}}^{V}, \equiv_{\mathrm{Aut}}^{V}, \equiv_{\mathrm{Con}}^{V}$ для основных классических многообразий груп, колец, решеток, булевых алгебр, не попавших в приведенную вьше таблицу, либо указанных в ней, но с открытьм вопросом об описании соответствуюших ему отношений.

В заключение приведем еще один результат, связанный с элементарной эквивалентностью производных структур свободных алгебр.

ТеОРема 5.13 (А. Г. Пинус [79]). Пусть $V$ - многообразие дистрибутивньх решеток и $\operatorname{Con}^{\prime} \mathscr{F}_{V}(k)$ есть частичная универсальная алгебра $\left\langle\operatorname{Con} \mathscr{F}_{V}(k) ; \vee, \wedge, \circ\right\rangle$, где о - частичная операчия на $\operatorname{Con} \mathscr{F}_{V}(k): \theta_{1} \circ \theta_{2}$ является произведением конгруэниий $\theta_{1}, \theta_{2}$, если $\theta_{1} \circ \theta_{2}$ есть конгруэнция на $\mathscr{F}_{V}(k)\left(\right.$ если $\theta_{1} \circ \theta_{2}=\theta_{2} \circ$ $\left.\theta_{1}=\theta_{1} \vee \theta_{2}\right)$, и не определена в противном случае. В этом случае отношение $\operatorname{Con}^{\prime} \mathscr{F}_{v}(k) \equiv \operatorname{Con}^{\prime} \mathscr{F}_{V}(\lambda)$ имеет место тогда и только тогда, когда $k \equiv_{2} \lambda$.

О близких к затронутым здесь вопросах см. также работу С. Шелаха [98].

\section{§ 6. Производные структуры топологических пространств}

В заключение коротко остановимся на результатах, связанных с приложением языка логики первого порядка для изучения топологических пространств. Значительная часть теории топологических пространств формализуется в рамках теории различных их производных структур, носящей чисто алгебраический характер. Наиболее часто в этой роли выступают алгебра замыканий $\mathfrak{A}(X)$, pешетка замкнутых подмно сеств $L(X)$, кольцо действительнозначных непрерывных функиий $C(X)$ топологического пространства $X$. Напомним, что $\mathfrak{A}(X)=\langle P(X) ; \cup, \cap, \neg, \varnothing, X,-\rangle$, где $\mathfrak{A}(X)=\langle P(X) ; \cup, \cap, \neg, \varnothing, X$,$\rangle - булева алгебра всех подмножеств множества X, \mathrm{a}^{-}$ - одноместная операция на $P(X)$ замькания (в пространстве $X$ ) подмножеств множества $X$. Решетка $L(X)$ и кольцо $C(X)$ определяются естественным образом. Заметим, что элементарная теория алгебры $\mathfrak{A}(X)$, очевидньм образом, включает в себя элементарную теорию решетки $L(X)$. В работе [62] доказано, что для класса так называемых совершенно нормальных пространств элементарная эквивалентность их колец непрерывных функций влечет элементарную эквивалентность решеток их замкнутых подмножеств. Изучение элементарных теорий подобных алгебраических про- 
изводных структур для топологических пространств начато в работе А. Гржегорчика [37], доказавшего неразрешимость элементарной теории класса $\mathfrak{A}(\Im)=\{\mathfrak{A}(X) \mid$ $X$ - произвольное топологическое пространство $\}$. Некоторая систематическая программа исследований элементарных теорий производных структур топологических пространств была предложена в работе [43]. Дальнейшее изучение элементарных теорий производных структур топологических пространств было, по большей части, связано с изучением элементарных теорий алгебраических производных при тех или иных ограничениях на класс рассматриваемых топологических пространств. Последнее, в частности, объяснимо тем, что абсолютно бесперспективны попытки построить элементарную классификацию производных структур для класса всех топологических пространств, ввиду столь же очевидной невозможности найти достаточно эффективную систему теоретико-множественных инвариантов отношения гомеоморфности для класса всех топологических пространств. Хотя для ряда частных случаев получены необходимые и достаточные условия элементарной эквивалентности производных структур произвольного пространства $X$ производным структурам того или иного фиксированного топологического пространства. Так, в работе Ю. Гуревича и С. Шелаха [40] найдены необходимые и достаточные условия на топологическое пространство $X$, при которых алгебра $\mathfrak{A}(X)$ элементарно эквивалентна алгебре $\mathfrak{A}(Q)$, где $Q$ - пространство рациональных чисел с порядковой топологией. Отметим также, что из результатов работы М. Рабина [87] вытекает утверждение следующей теоремы.

Теорема 6.1 (М. Рабин [87]). Элементарная теория алгебры $\mathfrak{A}(\mathbb{Q})$ разрешима.

Вообще, изучение элементарных теорий алгебр замьканий $\mathfrak{A}(X)$ топологических пространств $X$ с порядковой топологией, индуцированной некоторым отношением плотного линейного порядка $\leqslant$ на множестве $X$, довольно часто равносильно исследованию монадической теории второго порядка линейно упорядоченного множества $\langle X ; \leqslant\rangle$ (обе теории интерпретируются друг в друге). В частности, очевидньм образом, это имеет место для упорядоченного множества $\mathbb{R}$ действительных чисел. В работе С. Шелаха [97] доказана неразрешимость монадической теории упорядоченного множества $\langle\mathbb{R} ; \leqslant\rangle$, и, тем самьм, имеет место следуюшая теорема.

Теорема 6.2 (С. Шелах [97]). В предположсении континуум-гипотезы элементарная теория алгебрь $\mathfrak{A}(\mathbb{R})$ неразрешима.

В работах Ю. Гуревича [38], [39] утверждение этой теоремы перенесено на более широкий класс топологических пространств, включаюший в себя, в частности, и канторовский дисконтинуум.

Отметим, что из уже упомянутых выше результатов М. Рабина вытекает контрастное к утверждению теоремы 6.2 утверждение.

Теорема 6.3 (М. Рабин [87]). Элементарная теория решетки $L(\mathbb{R})$ разреши$\mathcal{M a}$.

Другим дизъюнктным к классу топологических пространств, близких к пространствам $\mathbb{R}$ и $\mathbb{Q}$, но также довольно тщательно изученньг, в рассматриваемом контексте, классом топологических пространств, является класс разреженных пространств. Напомним, что топологическое пространство $X$ назьвается разреженнылм, если оно не содержит совершенных подпространств, т.е. если последовательная итерация взятия 
производной Кантора-Бендиксона, начатая с пространства $X$, приводит к пустому множеству. При этом, в частности, доказаны следующие утверждения.

ТеОрема 6.4 (Г. Херре, Д. Зеезе [44]). В предположении существования слабо компактного кардинала әлементарная теория класса алгебр замыканий $T_{2}$-пространств с пустой второй производной неразрешима.

Теорема 6.5 (А.Г. Пинус [74]). а) Элементарная теория класса решеток замкнутых подмножсеств $T_{1}$-пространств с конечным числом предельных точек неразрешима.

б) Элементарная теория класса алгебр замыканий $T_{2}$-пространств с конечнылм числом предельных точек разрешима.

Заметим, что дополнительные (к ZFC) теоретико-множественные предположения в формулировках теорем $6.2,6.4$ возникают не случайно. Элементарные теории производных структур ряда топологических пространств не являются однозначно определенньми в рамках теоретико-множественной аксиоматики ZFC. К примеру, имеет место следующее утверждение.

Теорема 6.6 (Г. Херре, Д. Зеезе [44]). Существует әлементарная формула $\varphi$ сигнатуры алгебр замыкания, вхождение, либо не вхождение которой в элементарную теорию алгебры $\mathfrak{A}(X)$ не зависит от $Z F C$, здесь $X$ - пространство, определенное на ординале $\omega_{2}+1$ порядковой топологией.

В связи с отмеченной выше невозможностью построения элементарной классификации алгебраических производных структур для класса всех топологических пространств, естественно, возникает вопрос о доказательстве утверждений типа теоремы Левенгейма-Сколема для данного класса структур, а точнее, нахождение для данного класса структур чисел Левенгейма и Ханфа. Пусть $\mathfrak{A}(\Im)(L(\Im), C(\Im))$ - класс всех алгебр замыканий (класс всех решеток замкнутых подмножеств, класс всех колец непрерьвных функций соответственно) для произвольных топологических пространств.

ТеОрема 6.7 (А.Г. Пинус [73]). Числа Левенгейма (числа Ханфа) для классов $\mathfrak{A}(\Im)$ и L(ऽ) совпадают с числом Левенгейма (числом Ханфа) полной логики второго порядка.

Представляет интерес нахождение чисел Левенгейма и Ханфа для класса $C(\Im)$.

\section{СПИСОК ЛИТЕРАТУРЫ}

[1] Р. Бэр. Линейная алгебра и проективная геометрия. М.: ИЛ, 1955.

[2] J. T. Baldwin, S. Shelah. Second-order quantifiers and the complexity of theories // Notre Dame J. Formal Logic. 1985. V. 26. № 3. P. 229-303.

[3] Б. Баясгалан. Разрешимость теорий производных структур полугрупп // Алгебраические системы и их многообразия. Свердловск: Урал. гос. ун-т им. А. М. Горького, 1988. C. $4-13$.

[4] Б. Баясгалан. Ограниченные теории некоторых классов квазиупорядоченных множеств // Исследования алгебраических систем. Свердловск: Урал. гос. ун-т им. А.М. Горького, 1989. C. $23-30$.

[5] Б. Баясгалан. Разрешимые теории производных структур полугрупп // Автореферат дисс. ... канд. физ.-матем. наук. Свердловск, 1991.

[6] Б. Баясгалан. Разрешимость элементарных теорий решеток подполугрупп // Сиб. матем. журн. 1990. Т. 31. № 1. С. 27-33. 
[7] Б. Баясгалан, Ю. М. Важенин. О разрешимости теорий классов степеней полугрупп // Изв. вузов. Матем. 1988. № 11. С. 62-64.

[8] C. I. Beidar, A. V. Mikhalev. On Mal'cev's theorem on elementary equivalence of linear groups // Contemp. Math. 1992. V. 131, Part 1. P. 29-35.

[9] К.И. Бейдар, А. В. Михалев, Г. Е. Пунинский. Логические аспекты теории колец и модулей // Фундам. прикл. матем. 1995. Т. 1. № 1. С. 1-62.

[10] O. V. Belegradek. On the Mal'cev correspondence between rings and groups // Proceedings of the 7th Easter Conference on Model Theory. Berlin, 1989. P. 43-57.

[11] O.V. Belegradek. Groups elementarily equivalent to $\mathrm{UT}_{3}(R) / /$ Proceedings of the 8th Easter Conference on Model Theory. Berlin, 1990. P. 31-38. (Seminarberichte. V. 110.)

[12] O. V. Belegradek. The Mal'cev correspondence revisited // Contemp. Math. 1992. V. 131, Part 1. P. 37-59.

[13] О.В. Белеградек. Соответствие Мальцева и неразрешимость // Сиб. матем. журн. 1992. Т. 33. № 4. С. 24-29.

[14] О. В. Белеградек. Унитреугольные группы и неразрешимость // Изв. вузов. Матем. 1993. № 3. C. 19-22.

[15] O. V. Belegradek. Some open problems in the model theory of unitriangular groups // Proceedings of the 10th Easter Conference on Model Theory. Berlin: Humboldt Univ., 1993. P. 9-13.

[16] O. V. Belegradek. Quasi-unitriangular groups // J. Symbolic Logic. 1993. V. 58. № 1. P. $205-218$

[17] O. V. Belegradek. The model theory of unitriangular groups // Ann. Pure Appl. Logic. 1994. V. 68. № 3. P. 225-261.

[18] O. V. Belegradek. Model theory of unitriangular groups // Amer. Math. Soc. Transl. Ser. (2). 1999. V. 195. P. 1-116.

[19] O. V. Belegradek, V. Tolstykh. The logical complexity of theories associated with an infinite-dimensional vector space // Proceedings of the 9th Easter Conference on Model Theory. Berlin: Humboldt Univ., 1991. P. 12-34.

[20] R. Bonnet. Very strongly rigid Boolean algebras continuum discrete set condition, countable anti-chain condition // Algebra Universalis. 1980. V. 11. № 3. P. 9-25.

[21] Е.И. Бунина. Элементарная эквивалентность унитарных линейных групп // Фундам. прикл. матем. 1998. Т. 4. № 4. С. 1265-1278.

[22] Е. И. Бунина. Элементарная эквивалентность унитарных линейных групп над колшцами и телами // УМН. 1998. Т. 53. № 2. С. 137-138.

[23] Е.И. Бунина. Элементарная эквивалентность групп Шевалле // Труды седьмых математических чтений МГСУ. 2000. С. 120-124.

[24] Е. И. Бунина. Элементарная эквивалентность групп Шевалле // УМН. 2001. Т. 56. №1. C. 157-158.

[25] Е.И. Бунина, А. В. Михалев. Элементарная эквивалентность линейных и алгебраических групп // Фундам. прикл. матем. 2000. Т. 6. № 3. С. 707-722.

[26] S. Burris, H.P. Sankappanavar. Lattice-theoretic decision problems in universal algebra // Algebra Universalis. 1975. V. 5. № 2. P. 163-177.

$[27]$ В. Г. Дурнев. Неразрешимость некоторых теорий групп $S L(n, \mathbb{Z})$ и $G L(n, \mathbb{Z}) / /$ Вопросы теории групп и гомологической алгебры. Ярославль: Ярославский гос. университет, 1994. C. $61-68$.

[28] В. Г. Дурнев. Об элементарных теориях целочисленных линейных групп // Изв. РАН. Сер. матем. 1995. Т. 59. № 5. С. 41-58.

[29] ЖК. Дьёдонне. Геометрия классических групп. М.: Мир, 1974.

[30] Ю. Л. Ершов. Неразрешимость теорий симметрических и простых конечных групп // Докл. АН СССР. 1964. Т. 158. № 4. С. 777-779.

[31] Ю. Л. Ершов. Проблемы разрешимости и конструктивные модели. М.: Наука, 1980.

[32] Ю. Л. Ершов, И. А. Лавров, А. Д. Тайманов, М. А. Тайцлин. Элементарные теории // УМН. 1965. Т. 20. № 4. С. 37-108.

[33] Ю. Л. Ершов, Е. А. Палютин. Математическая логика. М.: Наука, 1987.

[34] Э.И. Фридман. Об универсалшных теориях решеток подгрупп групп // Алгоритмические вопросы алгебраических систем и ЭВМ. Иркутск, 1979. С. 172-176. 
[35] И. З. Голубчик, А. М. Михалев. Изоморфизмы общей линейной группы над ассоциативными кольцами // Вестник Моск. ун-та. Сер. І матем. мех. 1983. № 3. С. 61-72.

[36] И.З. Голубчик, А. В. Михалев. Изоморфизмы унитарных групп над ассоциативными кольцами // Записки научных семинаров ЛОМИ. 1983. Т. 132. С. 97-109.

[37] A. Grzegorczyk. Undecidability of some topological theories // Fund. Math. 1951. V. 38. № 1. P. 137-152.

[38] Y. Gurevich. Monadic theory of order and topology. I // Israel J. Math. 1977. V. 27. № 3-4. P. 299-319.

[39] Y. Gurevich. Monadic theory of order and topology. II // Israel J. Math. 1979. V. 34. № 1-2. P. 45-71.

[40] Y. Gurevich, S. Shelah. Modest theory of short chains. II // J. Symbolic Logic. 1979. V. 44. № 4. P. 491-502.

[41] L. Heindorf. Comparing the expressive power of some languages for boolean algebras // Z. Math. Logik Grundlag. Math. 1981. V. 27. № 5. P. 419-434.

[42] L. Heindorf. Regular ideals and Boolean pairs // Z. Math. Logik Grundlag. Math. 1984. V. 30. №6. P. 547-560.

[43] G. W. Henson, C. G. Jockusch, L. A. Rubel, G. Takeuti. First order topology // Dissertationes Math. (Rozprawy Mat.). 1977. V. 143. P. 5-40.

[44] H. Herre, D. Seese. Concerning the monadic theory of the topology of well-ordering and scattered spaces // Bull. Acad. Polon. Sci. Sér. Sci. Math. 1980. V. 28. № 1-2. P. 1-6.

[45] М. И. Каргаполов. Об элементарной теории структур подгрупп // Алгебра и логика. 1962. T. 1. №3. C. $46-53$.

[46] Г. Т. Козлов. Неразрешимость элементарных теорий решеток подгрупп конечных абелевых p-групп // Алгебра и логика. 1970. Т. 9. № 2. С. 167-171.

[47] А. И. Кокорин. Связь элементарной и подгрупповой теорий абелевых групп // Тезисы IV Всесоюзной конференции по математической логике. Кишинев, 1976. С. 65.

[48] А. И. Кокорин, А. Г. Пинус. Вопросы разрешимости расширенных теорий // УМН. 1978. Т. 33. № 2. С. 49-84.

[49] Ж. Лаллеман. Полугруппы и комбинаторные приложения. М.: Мир, 1985.

[50] L. Lipshitz. The undecidability of the word problems for projective geometries and modular lattices // Trans. Amer. Math. Soc. 1974. V. 193. № 1. P. 171-180.

[51] Н.Г. Луковников, В.И. Мартьянов. Универсальные элементарно-подгрупповые теории абелевых групп // Алгоритмические вопросы алгебраических систем / Под ред. А. И. Кокорина. Иркутск, 1978. С. 42-57.

[52] А. И. Мальцев. Об одном соответствии между кольцами и группами // Матем. сб. 1960. T. 50. №3. С. 257-266.

[53] А. И. Мальцев. Об элементарных свойствах линейных групп // Некоторые проблемы математики и механики. Новосибирск: Наука, 1961. С. 110-132.

[54] W. Marek. Consistance d'une hypothèse de Fraïssé sur la définissabilité dans un language du second ordre // C. R. Acad. Sci. Paris Sér. A-B. 1973. V. 276. P. A1147-A1150.

[55] W. Marek. Sur la consistance d'une hypothèse de Fraïssé sur la définissabilité dans un language du second ordre // C. R. Acad. Sci. Paris Sér. A-B. 1973. V. 276. P. A1169-A1172.

[56] В.И. Мартьянов. О теории абелевых групп и булевых алгебр с автоморфизмами // IV Всесоюзная конференция по математической логике. Кишенев, 1976.

[57] В.В. Маевский. Об ограниченных теориях бесконечных симметрических групп и полугрупп // Тезисы Международной конференции по алгебре, посвященной памяти А. И. Мальцева. Новосибирск, 1989. С. 73.

[58] В.В. Маевский. Критические теории бесконечных симметрических полугрупп // Тезисы Всесоюзного симпозиума по теории полугрупп. Свердловск: Уралшский гос. университет, 1988. С. 51.

[59] R. McKenzie. On elementary types of simmetric groups // Algebra Universalis. 1971. V. 1. № 1. P. 13-20.

[60] Е. М. Могилянская. Решение проблемы Тамуры // Современный анализ и геометрия. Ленинград: Ленинградский гос. педагогический университет, 1972. С. 148-151.

[61] V.A. Molchanov. Semigroups of mappings on graphs // Semigroup Forum. 1983. V. 27. №1. P. 155-199. 
[62] Ф.А.Мурзин. Об элементарной эквивалентности колец непрерывных функций // Теория моделей и ее приложения. Алма-Ата: Казахский гос. университет, 1980. С. 72-74.

[63] Ю.В. Нагребецкая. О границе разрешимости бесконечной симметрической группы // Изв. УрГУ. Матем. мех. 1999. № 14. С. 109-118.

[64] Ю. В. Нагребецкая. О разрешимости теорий первого порядка групп и моноидов целочисленных матриц // Алгебра и логика. 2000. Т. 39. № 4. С. 480-504.

[65] Ю. В. Нагребецкая. О граничной эквивалентности колец и матричных колец над ними // Алгебра и логика. 2000. Т. 39. №6. С. 693-710.

[66] Ю.В. Нагребецкая. О различимости ограниченных теорий целочисленных линейных групп // Труды международного семинара, посвященного 90-летию со дня рождения С. Н. Черникова. Екатеринбург: Уральский гос. университет, 2002. С. 180-183.

[67] Ю. В. Нагребецкая. О границах разрешимости колец целочисленных матриц // Деп. в ВИНИТИ. № 286, 2000.

[68] C. Naturman, H. Rose. Elementary equivalent pairs of algebras associated with sets // Algebra Universalis. 1991. V. 28. № 3. P. 324-338.

[69] А. Г. Пинус. Замечания к статье Ю. М. Важенина "Элементарные свойства полугрупп преобразований упорядоченных множеств" // Алгебра и логика. 1971. Т. 10. №3. C. $327-328$.

[70] А. Г. Пинус. О теории вьпуклых подмножеств // Сиб. матем. журн. 1972. Т. 13. № 1. C. $218-224$.

[71] A. G. Pinus. Elementary definability of symmetry groups // Algebra Universalis. 1973. V. 3. № 1. P. 59-66.

[72] А. Г. Пинус. Элиминируемость кванторов $Q_{0}$ и $Q_{1}$ на симметрических группах // Изв. вузов. Матем. 1979. № 12. С. 45-47.

[73] А.Г. Пинус. Об элементарной эквивалентности топологических пространств // Сиб. матем. журн. 1979. Т. 20. № 2. С. 433-439.

[74] А. Г. Пинус. Монадические теории разреженных топологических пространств и логики второго порядка // Алгоритмические вопросы алгебраических систем и ЭВМ. Иркутск: Изд-во ИрГУ, 1985. С. 50-59.

[75] А. Г. Пинус. Элементарная эквивалентность решеток разбиений // Сиб. матем. журн. 1988. T. 29. № 3. С. 211-212.

[76] А. Г. Пинус. Теории второго порядка конгруэнц-дистрибутивных многообразий // Алгебра и ее приложения. Иркутск: Иркутский гос. университет, 1994. С. 102-110.

[77] А. Г. Пинус. Об элементарных теориях генерических мултиалгебр // Изв. вузов. Матем. 1999. № 4. С. 39-43.

[78] А. Г. Пинус. Об определимости конечных алгебр производными структурами // Изв. вузов. Матем. 2001. № 4. С. 38-42.

[79] А. Г. Пинус. Об элементарной эквивалентности производных структур свободных решеток // Изв. вузов. Матем. 2002. № 5. С. 44-47.

[80] А. Г. Пинус. Условные термы и их приложения в алгебре и теории вычислений. Новосибирск: Изд-во НГТУ, 2002.

[81] А.Г. Пинус. Элементарная эквивалентность производных структур свободных полугрупп, унаров, групп // Алгебра и логика. 2004. Т. 43. №6. С. 731-748.

[82] А.Г. Пинус, З. А. Дулатова. Ограниченные теории решеток подалгебр некоторых хорновых классов // Изв. вузов. Матем. 1986. № 11. С. 79-81.

[83] A. G. Pinus, R.Sz. Madarász. On generic multi-algebras // Novi Sad J. Math. 1997. V. 27. № 2. P. 77-82.

[84] А. Г. Пинус, Г. Роуз. Элементарная эквивалентность решеток подалгебр свободных алгебр // Алгебра и логика. 2000. Т. 39. № 5. С. 595-601.

[85] A. G. Pinus, H. Rose. Second order equivalence of cardinals: an algebraic approach // Contributions to General Algebras 13. Klagenfurt: Heyn, 2001. P. 275-284.

[86] Б. И. Плоткин. Группы автоморфизмов алгебраических систем. М.: Наука, 1966.

[87] M. Rabin. Decidability of second-order theories and automata on infinite trees // Trans. Amer. Math. Soc. 1969. V. 141. № 1. P. 1-35.

[88] В.Н. Ремесленников, В.А. Романьков. Теоретико-модельные и алгоритмические вопросы теории групп // Итоги науки и техники. Сер. Алгебра. Топология. Геометрия. Т. 21. М.: ВИНИТИ, 1983. С. 3-79. 
[89] B. Rose. The $\aleph_{1}$-categoricity of strictly upper triangular matrix rings over algebraically closed fields // J. Symbolic Logic. 1978. V. 43. № 2. P. 250-259.

[90] M. Rubin. The theory of Boolean algebras with a distinguished subalgebras is undecidable // Ann. Sci. Univ. Clermont. 1976. № 60. Math. № 13. P. 129-134.

[91] M. Rubin. On the automorphism groups of homogeneons and saturated boolean algebras // Algebra Universalis. 1979. V. 9. № 1. P. 54-86.

[92] M. Rubin. On the automorphism groups of countable Boolean algebras // Israel J. Math. 1980. V. 35. № 1-2. P. 151-170.

[93] M. Rubin, S. Shelah. On the elementary equivalence of automorphism groups of Boolean algebras; downward Skolem-Löwenheim theorem and compactness of related quantifiers // J. Symbolic Logic. 1980. V. 45. № 2. P. 265-283.

[94] S. Shelah. Every two elementarily equivalent models have isomorphic ultrapowers // Israel J. Math. 1971. V. 10. № 2. P. 224-233.

[95] S. Shelah. There are just four second-order quantifiers // Israel J. Math. 1973. V. 15. № 2. P. $282-300$.

[96] S. Shelah. First order theory of permutation groups // Israel J. Math. 1973. V. 14. №1. P. 149-162; corrections: ibid. 1973. V. 15. № 3. P. 437-441.

[97] S. Shelah. The monadic theory of order // Ann. of Math. (2). 1975. V. 102. № 3. P. 379-419.

[98] S. Shelah. On what I do not understand (and have something to say), model theory // Math. Japon. 2000. V. 51. № 2. P. 329-377.

[99] S. Shelah. Interpreting set theory in the endomorphism semi-group of a free algebra or in a category // Ann. Sci. Univ. Clermont. 1976. № 60. Math. № 13. P. 1-29.

[100] Л.Н. Шеврин. Об элементарных структурных свойствах полугрупп // Докл. АН CCCP. 1966. T. 167. № 2. C. 305-308.

[101] Л.Н. Шеврин. Элементарные структурные свойства полугрупп // Сиб. матем. журн. 1966. T. 7. №3. C. $664-684$.

[102] Л.Н. Шеврин, А.Я. Овсянников. Полугруппы и их подполугрупповые решетки. Ч. 1: Полугруппы с некоторыми типами решеток подполугрупп и решеточные характеристики классов полугрупп. Свердловск: Уральский гос. университет, 1990.

[103] А. М. Слободской. Универсалшная теория группы $G L(3, \mathbb{Z})$ // Алгоритмические вопросы алгебраических систем и ЭВМ. Иркутск: Иркутский гос. университет, 1979. C. $200-217$.

[104] А. М. Слободской, Э. И. Фридман. Неразрешимые универсальные теории решеток подгрупп абелевых групп // Алгебра и логика. 1976. Т. 15. № 2. С. 227-234.

[105] М. Судзуки. Строение группы и строение структуры ее подгрупп. М.: ИЛ, 1960.

[106] Д. А. Супруненко. Группы матриц. М.: Наука, 1972.

[107] М.А. Тайцлин. Об элементарных теориях решеток подгрупп // Алгебра и логика. 1970. T. 9. № 4. C. $473-483$.

[108] A. Tarski. Arithmetical classes and types of Boolean algebras // Bull. Amer. Math. Soc. 1949. V. 55. № 1 . P. 64.

[109] В. А. Толстых. О некоторых интерпретациях в общей линейной группе // Труды советско-французского коллоквиума по теории моделей. Караганда: КарГУ, 1990. С. 202-214.

[110] V. Tolstykh. Set theory is interpretable in the automorphism group of an infinitely generated free group // J. London Math. Soc. (2). 2000. V. 62. № 1. P. 16-26.

[111] V. Tolstykh. Elementary equivalence of infinite-dimensional classical groups // Ann. Pure Appl. Logic. 2000. V. 105. № 1. P. 103-156.

[112] V. Tolstykh. The automorphism tower of a free group // J. London Math. Soc. (2). 2000. V. 61. № 2. P. 423-440.

[113] V. Tolstykh. On the logical strength of the automorphism groups of free nilpotent groups // Contemp. Math. 2002. V. 302. P. 113-120.

[114] V. Tolstykh. What does the automorphism group of a free Abelian group $A$ know about $A$ // Contemp. Math. (to appear).

[115] Ю. М. Важенин. Элементарные свойства полугрупп преобразований упорядоченных множеств // Алгебра и логика. 1970. Т. 9. № 3. С. 281-301.

[116] Ю. М. Важенин. Об элементарной определимости и элементарной характеризуемости классов рефлексивных графов // Изв. вузов. Матем. 1972. № 7. С. 3-17. 
[117] Ю. М. Важенин. О глобальной надполугруппе симметрической полугруппы // Математические записки УрГУ. 1974. Т. 9. № 1. С. 3-10.

[118] Ю. М. Важенин. Об элементарных теориях симметрических групп и полугрупп // Изв. вузов. Матем. 1974. № 1. С. 15-20.

[119] Ю. М. Важенин. Об элементарных теориях структур подполугрупп // Тезисы III Всесоюзной конференции по математической логике. Новосибирск: ИМ СО АН СССР, 1974. C. 24-25.

[120] Ю. М. Важенин. Элементарные свойства полугрупп частичных преобразований рефлексивных графов // Матем. записки УрГУ. 1977. Т. 10. № 3. С. 3-19.

[121] Ю. М. Важенин. Алгоритмические проблемы и иерархии языков первого порядка // Алгебра и логика. 1987. Т. 26. № 4. С. 419-434.

[122] Ю. М. Важенин. Алгоритмические проблемы и критические теории // УМН. 1987. T. 42. №3. C. $235-236$.

[123] Ю. М. Важенин. Критические теории // Сиб. матем. журн. 1988. Т. 29. № 1. С. 23-31.

[124] Ю. М. Важенин. Разрешимость теорий первого порядка классов полугрупп // Алгебраические системы и их многообразия. Свердловск: Урал. гос. ун-т им. А. М. Горького, 1988. C. $23-40$.

[125] Ю. М. Важенин, Е. В. Линецкий. Об элементарной теории решеток подполугрупп конечных полурешеток // Тезисы VII Всесоюзной конференции по математической логике. Новосибирск: ИМ СО АН СССР, 1984. С. 28.

[126] Ю. М. Важенин, Ю. В. Нагребецкая. Критические теории групп и моноидов целочисленных матриц // Изв. вузов. Матем. 1998. № 7. С. 77-79.

[127] Ю. М. Важенин, С.В.Сизый. Квазимногообразия эндомоделей // Изв. вузов. Матем. 1988. №2. С. 66-67.

[128] C. R. Videla. On the model theory of the ring NT $(n, R)$ // J. Pure Appl. Algebra. 1988. V. 55. №3. P. 289-302.

[129] W. Wheeler. Model theory of strictly upper triangular matrix rings // J. Symbolic Logic. 1980. V. 45. № 3. P. 455-463.

Новосибирский государственный технический университет

Поступила в редакцию

E-mail: algebra@nstu.ru

28.05.2004 